\title{
Free In-Plane Vibration Analysis of Circular, Annular, and Sector Plates Using Isogeometric Approach
}

\author{
Xiaohui Qin, ${ }^{1}$ Guoyong Jin $\mathbb{D D}^{2}{ }^{2}$ Mingfei Chen, ${ }^{2}$ and Shaoping Yin ${ }^{1}$ \\ ${ }^{1}$ CSIC-705th Research Institute, Xi'an 710077, China \\ ${ }^{2}$ College of Power and Energy Engineering, Harbin Engineering University, Harbin 150001, China \\ Correspondence should be addressed to Guoyong Jin; guoyongjin@hrbeu.edu.cn
}

Received 11 November 2017; Revised 22 February 2018; Accepted 29 March 2018; Published 7 May 2018

Academic Editor: Vadim V. Silberschmidt

Copyright (C) 2018 Xiaohui Qin et al. This is an open access article distributed under the Creative Commons Attribution License, which permits unrestricted use, distribution, and reproduction in any medium, provided the original work is properly cited.

\begin{abstract}
The in-plane free vibration of sector, annular, and circular plates is investigated by isogeometric finite element approach on the basis of nonuniform rational B-spline (NURBS) basis functions. Under the current framework, both the displacement field and geometry of the sector, annular, and circular plates are modeled by NURBS basis functions to bridge the gap between the design of geometry and the analysis of variable field. The NURBS basic functions can not only preserve the exact geometry of sector, annular, and circular plates, but also provide higher continuity of basis function and its derivatives. The governing equations can be derived by employing the principle of virtual work and the desired solutions are obtained by using the Arnoldi Method. Several numerical examples of sector, annular, and circular plates are performed, and three refinement schemes (the $h$-, $p$-, and $k$-refinement strategies) are applied to demonstrate the convergence. Then the effectiveness and accuracy of the proposed approach are validated through comparisons with results obtained from the finite element analysis as well as open available literature. On this basis, some new numerical results of frequency parameters with mode shapes are shown and may be used as benchmark results for the vibration investigation in the future. In addition, the effects of sector angles and ratio of inside to outside radii on the in-plane vibration of sector, annular, and circular plates under different boundary conditions are fully demonstrated.
\end{abstract}

\section{Introduction}

The sector, annular, and circular plates are typical structural components used in engineering widely. The flexural vibration of sector, annular, and circular plates has been studied by researchers and design engineers with considerable interest. A number of investigations [1-5] have been devoted to flexural vibrations of structures, maybe because the flexural vibration has lower resonant frequencies and a decisive role in terms of fluid-structure coupling. However, the vibrations of structures also contain in-plane parts, which often appear in high frequency motions and large coupled structures. When the sound radiation and energy transmission of coupled structures are considered, the importance of in-plane vibration is nonnegligible. Consequently, it is of great significance to obtain deep in-plane vibration comprehensions of sector, annular, and circular plates.

Xing and Liu [6] applied a Rayleigh quotient variational principle to study the free in-plane vibration for rectangular plate. In the paper, all classical boundary restraints which included two various kinds of simple supports were taken into consideration. Bercin and Langley [7] analyzed the inplane vibration problem of plate structures by adopting the dynamic stiffness technique and classical finite element assembled technique. Nefovska-Danilovic and Petronijevic [8] also used this method to investigate the in-plane free vibration of arbitrarily restrained isotropic rectangular plate. Gorman [9] used the method of superposition to solve the problem of free in-plane vibration of plates; then he used this method to solve in-plane vibration of Levy-type plates [10] which have a pair of simply supported opposite edges at least. Andrianov et al. [11] adopted homotopy perturbation method to study the in-plane vibration of rectangular plate. Chen et al. [12] applied Chebyshev-Lagrangian approach to study in- and out-of-plane vibration of plate with cutout. Mohazzab and Dozio [13] used the spectral collocation method to analyze inplane vibration of isotropic skewed geometrical plates. Some 
other efforts [14-18] have also been devoted to the in-plane vibration problem of plate structures.

It is needed to point out that a great majority of existing investigations in aforementioned issues are restricted within rectangular plates. The related work about the inplane vibration of sector, annular, and circular plates is very limited. Irie et al. [19] studied the natural in-plane vibration frequencies for annular and circular plates with clamped and free boundary conditions by using the transfer matrix method. The Rayleigh-Ritz method was employed by Bashmal et al. [20] to investigate the natural in-plane vibration characteristics for circular disk. Farag and Pan [21] assumed that the in-plane vibration mode shapes in circumferential and radial direction were, respectively, expressed in a series summation of trigonometric functions and Bessel functions, and then they solved the in-plane vibration equations for the clamped circular plates. It is well known that differential quadrature method (DQM) as one of point discretization methods can solve this vibration. With this DQM, Eftekhari [22] investigated in-plane vibration of circular arches. In this investigation variable thickness and a moving point load are taken into consideration. Singh and Muhammad [23] solved free in-plane vibration of isotropic rectangular, rhombic, and annular sector plates with the finite element analysis. In their paper, the natural coordinates with multiple points were used to map the geometry. Chan [24] calculated in-plane vibration frequency of circular plate structures by utilizing Hamilton's principle. Shi et al. [25] calculated the natural in-plane vibration of annular sector plate with using the modified Fourier series method. This method was used, lately, by Wang et al. [26] to analyze the free in-plane vibration of orthotropic sector, annular, and circular plates.

The finite element method (FEM) is one of main numerical approaches to solve structural vibration. Under this method, the basis functions in traditional FEM are employed to describe the unknown structures' fields. In engineering occasions, however, some drawbacks of FEM may be encountered when obtaining a discretized geometric model, such as time-consuming refinement and cumbersome mesh generation, as well as with geometrical net errors. To overcome the gap between FEM and Computer Aided Design (CAD), recently Hughes et al. [27] introduced an isogeometric analysis (IGA) based on the NURBS basis functions [28]. In the IGA method, the geometry and the variable fields of structures are constructed by using the NURBS basis functions. These functions can preserve exact geometry and provide higher order approximations than Lagrange functions used in standard FEM. What is more, the refinement and meshing process may be implemented without CAD geometry.

In recent decades, the IGA method has been also applied to solve several mechanics problems, such as solid, fluid, contact, and structural mechanics [29-33]. Cho and $\mathrm{Ha}$ [34] investigated the exact geometry and enhanced sensitivity of shape design optimization. Wall et al. [35] applied this approach to solve structural shape optimization about twodimensional problems of linear elasticity. Based on patientspecific vascular NURBS modeling, Zhang et al. [36] used the isogeometric approach to analyze the flow of blood. Bazilevs et al. [37] analyzed the fluid-structure interaction problem about blood flow with the same theory. Kruse et al. [38] analyzed large deformation elasticity by isogeometric collocation methods; then they also solved frictional contact problems. Recently, the IGA was extended to study static and dynamic problems of various kinds of plate and shell structures. Isogeometric Kirchhoff-Love shell formulations were used by Kiendl et al. [39] to solve general hyperelastic materials. This method then was used by Tepole et al. [40] to analyze biological membranes. Benson et al. [41] used IGA method to study Reissner-Mindlin shell. What is more, the IGA method has been utilized in analyzing the vibration of rotation-free shells [42] and functionally graded and multilayered plates with various theories, such as higher order shear deformation [43-45], layerwise deformation [46], and new inverse trigonometric shear deformation theories [47]. Although a large number of studies on the basis of IGA method have been published, to the authors' knowledge, the works regarding the use of this method for the inplane vibration of sector, annular, and circular plates are still unavailable in the literature.

In this work, an approximate formulation based on NURBS functions is carried out for studying the in-plane vibration characteristics of the sector, annular, and circular plates, in which the CAD basis functions are utilized for approximating field variables. The principle of virtual work is used to derive the differential equations for free in-plane vibration of sector plate, annular plate, circular sector plate, and circular plate. The convergence study of the formulation is testified in several selected examples. Several numerical results of the natural in-plane frequency parameters for these plates are given. By comparing numerical results with data obtained by the FEM and existing literature, the convergence and reliability of the formulation are tested in several selected examples. In addition, the influence with regard to some effects on the frequency parameters of these plates and their associated mode shapes is also exerted, such as sector angles, the ratios between inner and outer radii, and different boundary conditions.

\section{Theoretical Formulations}

2.1. A Brief of NURBS Functions. A group of nondecreasing values $\xi_{i} \in R, i=1,2,3 \cdots m+p+1$, are used to define the knot vector $E=\left\{\xi_{1}, \xi_{2} \ldots \xi_{n+p+1}\right\}$. B-spline basis function is piecewise constant when the order of basis functions $p$ is zero.

$$
N_{i, 0}(\xi)= \begin{cases}1, & \xi_{i} \leq \xi \leq \xi_{i+1} \\ 0, & \xi_{i} \geq \xi, \quad \xi \geq \xi_{i+1} .\end{cases}
$$

For $p>0$, the basis functions can be calculated recursively as

$$
\begin{aligned}
N_{i, p}(\xi)= & \frac{\xi-\xi_{i}}{\xi_{i+p}-\xi_{i}} N_{i, p-1}(\xi) \\
& +\frac{\xi_{i+p+1}-\xi}{\xi_{i+p+1}-\xi_{i+1}} N_{i+1, p-1}(\xi),
\end{aligned}
$$

where the assumption $0 / 0=0$ is adopted. $m$ and $p$, respectively, are the total number and polynomial order of basic 


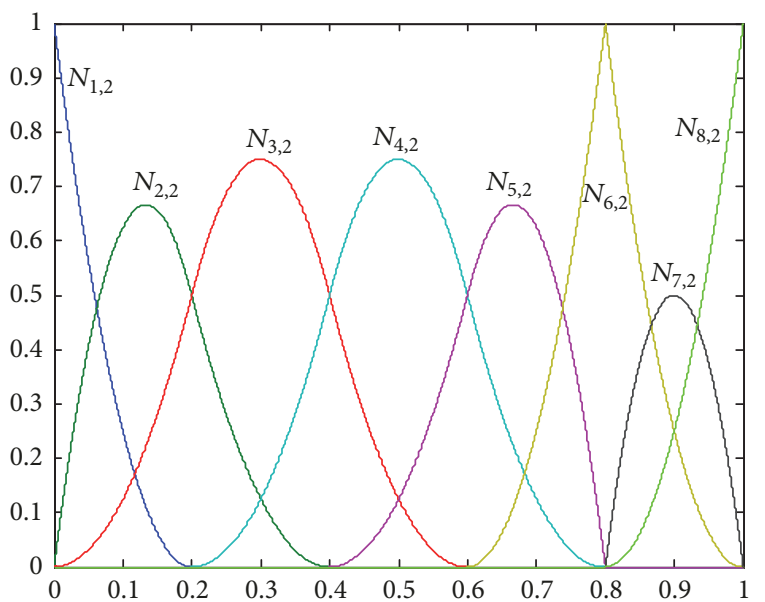

FIgURE 1: Quadratic basis functions for the knot vector $E=$ $\{0,0,0,1,2,3,4,4,5,5,5\}$.

functions. If there are $p+1$ same boundary knots in a knot vector, this vector belongs to the type of open knot vector. For the continuity of the basis function, it is easily found that the basis functions are $C^{p-k}$ continuous at the knot having multiplicity $k$ and $C^{p-1}$ continuous at nonrepeated knots. By employing the knot vector $E=\{0,0,0,1,2,3,4,4,5,5,5\}$, a series of quadratic B-spline basis functions can be easily obtained in Figure 1. It is obvious that these basis functions have $C^{0}$ continuity at the location of ends of the knot vector with repeated knot $\xi=0.8$ and $C^{1}$ continuity at the other nonrepeated knots.

However, it is insufficient to construct exactly arbitrary geometries only by B-spline basis functions, such as ellipse and circle. NURBS basis functions can overcome these limitations by introducing the weights to construct a great variety of geometrical entities. One-dimensional (1D) NURBS basis function of $p$ order can be obtained:

$$
R_{i, p}(\xi)=\frac{\omega_{i} N_{i, p}(\xi)}{\sum_{i=1}^{n} \omega_{i} N_{i, p}(\xi)},
$$

where $\omega_{i}$ is the weight referred to the $i$ th B-spline basis function $N_{i, p}(\xi)$.

Analogously, the 2D NURBS basis functions of orders $p$ and $q$ can be defined as

$$
R_{i, j}^{p, q}(\xi, \eta)=\frac{\omega_{i, j} N_{i, p}(\xi) M_{j, p}(\eta)}{\sum_{i=1}^{m} \sum_{j=1}^{n} \omega_{i, j} N_{i, p}(\xi) M_{j, q}(\eta)},
$$

where $M_{j, p}(\eta)$ is the $j$ th B-spline basis function in the $\eta$ direction. $\omega_{i, j}$ is the corresponding weight. $m$ and $n$ are the numbers of total basic functions in the $\xi$ - and $\eta$-direction, respectively.

Therefore, by giving a set of control points $p_{i, j}=\left\{x_{i, j}, y_{i, j}\right\}$ and 2D NURBS basis functions $R_{i, j}^{p, q}(\xi, \eta)$, a surface is written as

$$
S(\xi, \eta)=\sum_{i=1}^{m} \sum_{j=1}^{n} R_{i, j}^{p, q}(\xi, \eta) p_{i, j}
$$

For example, the exact geometries of annular sector plate, annular plate, circular sector plate, and circular plate with their corresponding control nets are shown in Figure 2.

Then, by employing the NURBS basis functions, the geometry coordinate ( $x$ and $y$ ) and the in-plane displacement fields $(u$ and $v$ ) of structures can be described as follows:

$$
\begin{aligned}
x(\xi, \eta) & =\sum_{a=1}^{n \times m} R_{a}(\xi, \eta) x_{a}, \\
y(\xi, \eta) & =\sum_{a=1}^{n \times m} R_{a}(\xi, \eta) y_{a} \\
u(\xi, \eta, t) & =\sum_{a=1}^{n \times m} R_{a}(\xi, \eta) u_{a}, \\
v(\xi, \eta, t) & =\sum_{a=1}^{n \times m} R_{a}(\xi, \eta) v_{a},
\end{aligned}
$$

where $R_{a}(\xi, \eta)=R_{i, j}^{p, q}(\xi, \eta)$ is the NURBS basis function, subscript $a=(i-1) \cdot n+j$ is used for convenience, $x_{a}$ and $y_{a}$ are the control point coordinates corresponding to relational basis function, and $\left(u_{a}, v_{a}\right)$ represents the displacement vector of the corresponding control points.

2.2. Description of the Models. The investigative geometry models for free in-plane vibration of the sector, annular, and circular plates in natural coordinates are presented in Figure 3. In following analysis, the materials of these sector plates are defined as isotropic with Poisson's ratio $v$, mass density $\rho$, and Young's modulus $E$. The thickness, inner radius, and outer radius of the annular plate sector are $h, R_{0}$, and $R_{1}$, respectively. All annular plate, circular sector plate, and circular plate can be obtained from the annular sector plate. It is required that the sector angle $\phi$ can be set to $2 \pi$ for the case of annular plate. Similarly, for the circular sector plate, it is required that the inner radius $R_{0}$ can be set to zero, while for the circular plate the sector angle $\phi$ and inner radius $R_{0}$ should be, respectively, set to $2 \pi$ and zero. However, in the coupling edges $\phi=0, \phi=2 \pi$ and inner radius $R_{0}$ $=0$, the kinematic and physical compatibility conditions of the above described annular plate, circular sector plate, and circular plate should be taken into consideration. To satisfy this characteristic, a scheme for coupling coincident control points analogous to coupling nodes in the finite element analysis is used to couple control points and nodes in the model.

2.3. Constitutive and Kinematic Relations. The stress-strain relations for the aforementioned isotropic sector, annular, and circular plates undergoing in-plane vibration can be obtained on the basis of generalized Hooke's law, expressed in the matrix form as

$$
\left\{\begin{array}{c}
\sigma_{x} \\
\sigma_{y} \\
\tau_{x y}
\end{array}\right\}=\left[\begin{array}{lll}
Q_{11} & Q_{12} & Q_{16} \\
Q_{12} & Q_{22} & Q_{26} \\
Q_{16} & Q_{26} & Q_{66}
\end{array}\right]\left\{\begin{array}{c}
\varepsilon_{x} \\
\varepsilon_{y} \\
\gamma_{x y}
\end{array}\right\},
$$



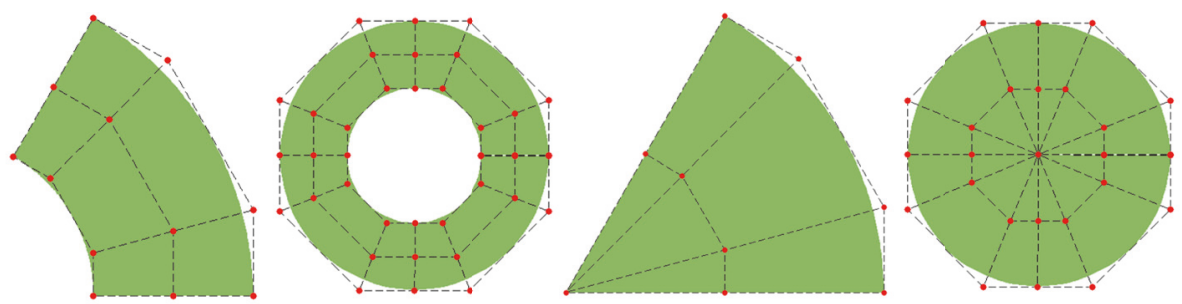

FIGURE 2: The exact geometries and control nets for sector plates of various types.

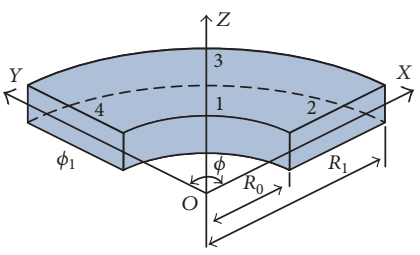

(a)

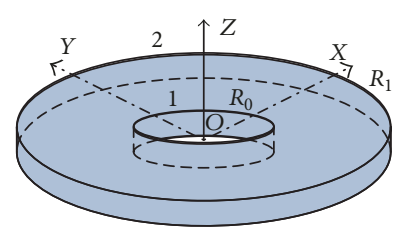

(b)

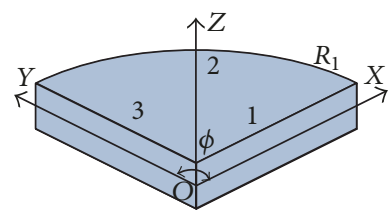

(c)

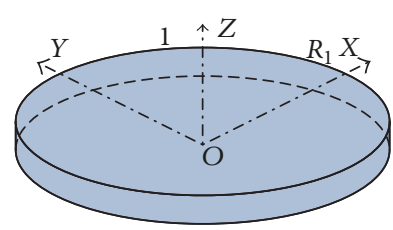

(d)

FIGURE 3: Isotropic annular sector plates of various types: (a) annular sector plate; (b) annular plate; (c) circular sector plate; (d) circular plate.

where $\sigma_{x}, \sigma_{y}, \tau_{x y}$ and $\varepsilon_{x}, \varepsilon_{y}, \gamma_{x y}$, respectively, are the stresses and strains. The elastic stiffness coefficients $Q_{i j}(i, j=1,2,6)$ are given as follows: $Q_{11}=Q_{22}=E /\left(1-\nu^{2}\right) ; Q_{12}=E v /\left(1-\nu^{2}\right)$; $Q_{16}=Q_{26}=0$; and $Q_{66}=E /(2(1+\nu))$.

Referring to the work of Singh and Muhammad [23], the in- and out-of-plane motions of these plates can be separated; therefore, the motions of these sector plates are assumed without out-of-plane part. Under this assumption the straindisplacement relations are written:

$$
\begin{aligned}
\varepsilon_{x} & =\frac{\partial u}{\partial x}, \\
\varepsilon_{y} & =\frac{\partial v}{\partial y}, \\
\gamma_{x y} & =\frac{\partial u}{\partial y}+\frac{\partial v}{\partial x} .
\end{aligned}
$$

In this paper, only the in-plane displacement components $(u$ and $v$ ), rather than the out-of-plane one $(w)$, are involved in the formulation. Therefore, the current formulation is suitable for the plates composed of the symmetric materials with respect to the mid-plane, such as single-layer isotropic, transverse isotropic, and orthotropic plates, as well as symmetric multilayered composite plates.

2.4. Solution Procedure. The potential $(U)$ energy of sector plate and the kinetic $(T)$ energy of sector plate are written as

$$
\begin{gathered}
U=\frac{1}{2} \iint_{S}\left(Q_{11} \varepsilon_{x}^{2}+2 Q_{12} \varepsilon_{x} \varepsilon_{y}+Q_{22} \varepsilon_{y}{ }^{2}+Q_{66} \gamma_{x y}{ }^{2}\right) h d x d y \\
T=\frac{1}{2} \iint_{S} \rho h\left((\dot{u})^{2}+(\dot{v})^{2}\right) d x d y,
\end{gathered}
$$

where $\rho, h$, and $S$ are, respectively, the mass density, thickness, and integral surface of sector plate, $\dot{u}=\partial u / \partial t$ and $\dot{v}=\partial v / \partial t$, respectively, represent in-plane velocity field components along the $x, y$ directions. The stress, strain components, and elastic stiffness coefficients are determined by (7) and (8).

By applying Hamilton's principle [48], the principle of virtual work for sector plate is expressed as

$$
\delta \int_{t_{1}}^{t_{2}}(U+T) d t=0
$$

Substituting (9)-(10) into (11), the weak statement of inplane vibration can been obtained as follows:

$$
\begin{aligned}
& \int_{t_{1}}^{t_{2}} \iint_{S}\left\{\left(Q_{11} \frac{\partial u}{\partial x}+Q_{12} \frac{\partial v}{\partial y}\right) \delta\left(\frac{\partial u}{\partial x}\right)\right. \\
& +\left(Q_{12} \frac{\partial u}{\partial x}+Q_{22} \frac{\partial v}{\partial y}\right) \delta\left(\frac{\partial v}{\partial y}\right)+Q_{66}\left(\frac{\partial u}{\partial y}+\frac{\partial v}{\partial x}\right) \\
& \quad \cdot \delta\left(\frac{\partial u}{\partial y}\right)+Q_{66}\left(\frac{\partial u}{\partial y}+\frac{\partial v}{\partial x}\right) \delta\left(\frac{\partial v}{\partial x}\right) \\
& \quad-\rho((\dot{u}) \delta(\dot{u})+(\dot{v}) \delta(\dot{v}))\} d x d y d t \\
& \quad=0
\end{aligned}
$$

The in-plane vibration governing equations of sector plate can be written by variable separation approach:

$$
\begin{aligned}
& Q_{11} \frac{\partial^{2} u}{\partial x^{2}}+\left(Q_{12}+Q_{66}\right) \frac{\partial^{2} v}{\partial x \partial y}+Q_{66} \frac{\partial^{2} u}{\partial y^{2}}=\rho \ddot{u} \\
& Q_{22} \frac{\partial^{2} v}{\partial y^{2}}+\left(Q_{12}+Q_{66}\right) \frac{\partial^{2} u}{\partial x \partial y}+Q_{66} \frac{\partial^{2} v}{\partial x^{2}}=\rho \ddot{v} .
\end{aligned}
$$


And the following definitions of stress resultants are used:

$$
\begin{aligned}
& \bar{N}_{x}=Q_{11} \frac{\partial u}{\partial x}+Q_{12} \frac{\partial v}{\partial y}, \\
& \bar{N}_{x y}=Q_{66}\left(\frac{\partial u}{\partial y}+\frac{\partial v}{\partial x}\right) \\
& \quad \text { at } r=R_{0}, R_{1} \\
& \bar{N}_{y}=Q_{12} \frac{\partial u}{\partial x}+Q_{22} \frac{\partial v}{\partial y}, \\
& \bar{N}_{y x}=Q_{66}\left(\frac{\partial u}{\partial y}+\frac{\partial v}{\partial x}\right) \\
& \text { at } \phi=0, \phi_{1},
\end{aligned}
$$

where $\bar{N}_{x}$ and $\bar{N}_{x y}$ are stress resultants in the $x$ - and $y$-axis directions in the radial edges, respectively. Similarly, $\bar{N}_{y}$ and $\bar{N}_{y x}$ are stress resultants in the $y$ - and $x$-axis directions in circumferential edges. [49]):

The boundary conditions are of the following form (see

$$
\begin{aligned}
& \bar{N}_{x} \text { or } u \\
& \bar{N}_{x y} \text { or } v \\
& \bar{N}_{y} \text { or } v \\
& \bar{N}_{y x} \text { or } u .
\end{aligned}
$$

According to the study of Cottrell [50], the IGA is analogous to the traditional FEM for using the homogeneous Dirichlet boundary conditions on $u, v$. The following boundary conditions could be used in this paper:

Free (F):

$$
\begin{aligned}
& \bar{N}_{x}=0, \\
& \bar{N}_{x y}=0 \\
& \quad \text { at } r=R_{0}, R_{1} \\
& \bar{N}_{y}=0, \\
& \bar{N}_{y x}=0 \\
& \text { at } \phi=0, \phi_{1} .
\end{aligned}
$$

Clamped (C):

$$
u=v=0 .
$$

As for the center of circular and circular sector plates and the circumferential edges of annular and circular plates, it is essential to consider their physical compatibility and kinematic conditions. Therefore, similar to standard FEM for coupling coincident nodes, IGA method can couple relevant
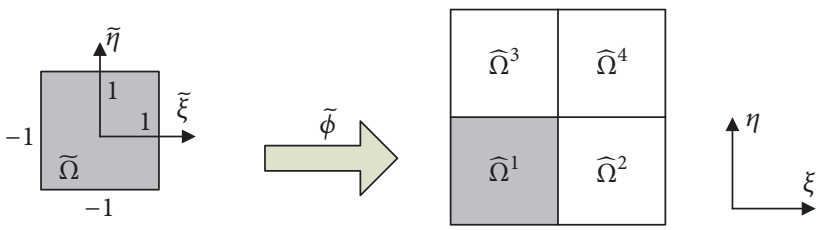

Parent space

Parameter space

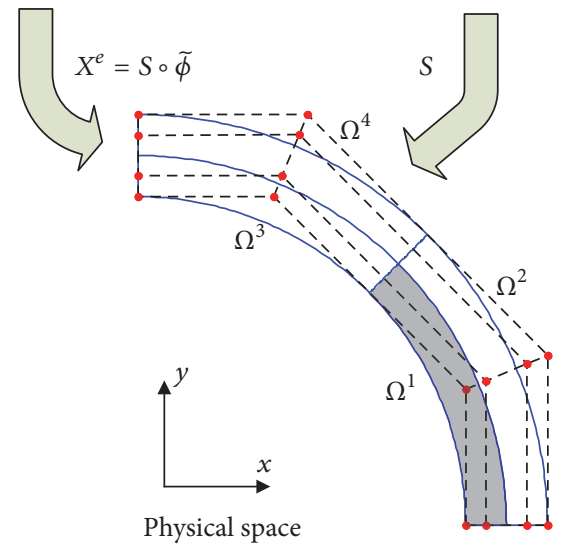

FIgURE 4: The mappings among parent space, parametric space, and physical space.

control points. From Figure 2, there are six control points in coupling edges $\phi=0$ of annular plate and four control points in the center of circular sector plate in original one. After coupling relevant control points, there are three control points and one point in coupling edges $\phi=0$ of annular plate and the center of circular sector plate, respectively.

The NURBS functions substitute for interpolation functions which are utilized in FEM to construct the unknown fields. A typical element for two-dimensional structures is used in the isogeometric analysis. There are present space for calculating integral equation and physical space in conventional finite element implementations, only. The parametric space is unnecessary. Figure 4 shows the parent, parametric, and physical spaces and mapping variations. The current space is mapped to geometrical space by $X^{e}=S \circ \widetilde{\phi}$, where mappings $\widetilde{\phi}: \widetilde{\Omega} \rightarrow \widehat{\Omega}$ and $S: \widetilde{\Omega} \rightarrow \Omega$, respectively, are from current space $\widetilde{\Omega}$ to parametric space $\widetilde{\Omega}$ and from parametric space $\widetilde{\Omega}$ to geometrical space $\Omega$.

An example is presented to explain the mapping $\widetilde{\phi}: \widetilde{\Omega} \rightarrow$ $\widehat{\Omega}$ for the $\widehat{\Omega}^{e}=\left[\xi_{i}, \xi_{i+1}\right] \otimes\left[\eta_{j}, \eta_{j_{+1}}\right]$ parametric space of an element

$$
\widetilde{\phi}^{e}\left\{\begin{array}{l}
\xi \\
\eta
\end{array}\right\}=\left\{\begin{array}{c}
\frac{1}{2}\left(\xi_{i+1}-\xi_{i}\right) \xi+\frac{1}{2}\left(\xi_{i+1}+\xi_{i}\right) \\
\frac{1}{2}\left(\eta_{j+1}-\eta_{j}\right) \eta+\frac{1}{2}\left(\eta_{j+1}+\eta_{j}\right)
\end{array}\right\}
$$

and the corresponding Jacobian determinant

$$
|\widetilde{J}|=\frac{1}{4}\left(\xi_{i+1}-\xi_{i}\right)\left(\eta_{j+1}-\eta_{j}\right) .
$$


According to (6), it is easy to calculate the mapping $S$ : $\widehat{\Omega} \rightarrow \Omega$. And the Jacobian of the relevant transformation is given:

$$
\widehat{J}=\left[\begin{array}{ll}
\frac{\partial x}{\partial \xi} & \frac{\partial x}{\partial \eta} \\
\frac{\partial y}{\partial \xi} & \frac{\partial y}{\partial \eta}
\end{array}\right]
$$

By using the first derivatives of NURBS basis functions, the Jacobian may be represented by the following expression:

$$
\widehat{J}=\left[\begin{array}{ll}
\sum_{a}^{n_{e}} \frac{\partial R_{a}}{\partial \xi} x_{a}^{e} & \sum_{i}^{n_{e}} \frac{\partial R_{a}}{\partial \eta} x_{a}^{e} \\
\sum_{a}^{n_{e}} \frac{\partial R_{a}}{\partial \xi} y_{a}^{e} & \sum_{a}^{n_{e}} \frac{\partial R_{a}}{\partial \eta} y_{a}^{e}
\end{array}\right],
$$

where $n_{e}$ is the number of total control points of each element. Therefore, the Jacobian $|\widehat{J}|$ can be determined by (21).

Considering the above Jacobian determinant $|\widehat{J}|$ and $|\widetilde{J}|$, the Jacobian determinant can be written as

$$
|J|=|\widehat{J}| \cdot|\widetilde{J}|
$$

In order to facilitate the analysis, the transformation formula which is used for interpretation of a quadrature rule from geometrical space to current space is written as

$$
\begin{aligned}
\iint_{S} f(x, y) d x d y & =\sum_{e}^{N_{\text {el }}} \iint_{\Omega^{e}} f(x, y) d x d y \\
& =\sum_{e}^{N_{\text {el }}} \iint_{\widetilde{\Omega}^{e}} f(\widetilde{\xi}, \widetilde{\eta})|J| d \widetilde{\xi} d \widetilde{\eta},
\end{aligned}
$$

where $N_{\mathrm{el}}$ is the total number of elements. It is easy to see that transformation formula can be obtained with Jacobian determinant and the mappings in Figure 4.

By using the separation of variables $u(x, t)=\tilde{u}(x) e^{j \omega_{l} t}$ and $v(x, t)=\widetilde{v}(x) e^{j \omega_{l} t}$, the principle of virtual work for sector plate in (10) can be written as

$$
\begin{aligned}
U & =\frac{1}{2} \iint_{S} h\left\{Q_{11}\left(\frac{\partial \widetilde{u}}{\partial x}\right)^{2}+2 Q_{12}\left(\frac{\partial \widetilde{v}}{\partial y}\right)\left(\frac{\partial \widetilde{u}}{\partial x}\right)\right. \\
& \left.+Q_{22}\left(\frac{\partial \widetilde{v}}{\partial y}\right)^{2}+Q_{66}\left(\frac{\partial \widetilde{u}}{\partial y}+\frac{\partial \widetilde{v}}{\partial x}\right)^{2}\right\} d x d y \\
T & =-\frac{1}{2} \iint_{S} \omega_{l}^{2} \rho h\left(\widetilde{u}^{2}+\widetilde{v}^{2}\right) d x d y,
\end{aligned}
$$

where $\omega_{l}$ is the $l$ th free vibration frequency of the sector plate.
The Lagrange function of sector plate can be written as

$$
\begin{aligned}
L= & \frac{1}{2} \iint_{S} h\left\{Q_{11}\left(\frac{\partial \widetilde{u}}{\partial x}\right)^{2}+2 Q_{12}\left(\frac{\partial \widetilde{v}}{\partial y}\right)\left(\frac{\partial \widetilde{u}}{\partial x}\right)\right. \\
& +Q_{22}\left(\frac{\partial \widetilde{v}}{\partial y}\right)^{2}+Q_{66}\left(\frac{\partial \widetilde{u}}{\partial y}+\frac{\partial \widetilde{v}}{\partial x}\right)^{2} \\
& \left.-\omega_{l}^{2} \rho\left(\widetilde{u}^{2}+\widetilde{v}^{2}\right)\right\} d x d y .
\end{aligned}
$$

The variables and geometry coordinate of control points in (6) can be written as

$$
\begin{aligned}
& x(\xi, \eta)=\sum_{a=1}^{n \times m} R_{a}(\xi, \eta) x_{a}, \\
& y(\xi, \eta)=\sum_{a=1}^{n \times m} R_{a}(\xi, \eta) y_{a} \\
& \tilde{u}(\xi, \eta)=\sum_{a=1}^{n \times m} R_{a}(\xi, \eta) \tilde{u}_{a}, \\
& v(\xi, \eta)=\sum_{a=1}^{n \times m} R_{a}(\xi, \eta) \widetilde{v}_{a} .
\end{aligned}
$$

Similarly, the geometry coordinate and the in-plane displacement fields of each element can be written as

$$
\begin{aligned}
& x^{e}(\xi, \eta)=\sum_{a=1}^{n_{e}} R_{a}(\xi, \eta) x_{a}^{e}, \\
& y(\xi, \eta)=\sum_{a=1}^{n_{e}} R_{a}(\xi, \eta) y_{a}^{e}, \\
& \widetilde{u}^{e}(\xi, \eta)=\sum_{a=1}^{n_{e}} R_{a}(\xi, \eta) u_{a}^{e}, \\
& \widetilde{v}^{e}(\xi, \eta)=\sum_{a=1}^{n_{e}} R_{a}(\xi, \eta) \widetilde{v}_{a}^{e},
\end{aligned}
$$

where $n_{e}$ is the number of total control points of each element; the superscript $e$ denotes use for each element.

By substituting (26) into (25) and then applying RayleighRitz procedure to solve every $\widetilde{u}_{a}$ and $\widetilde{v}_{a}$,

$$
\delta L=0 .
$$

According to the element assembly operator in the standard FEM and quadrature rule in (23), (27) can be also written as

$$
\begin{aligned}
\delta & \left\{\frac{1}{2} \sum_{e=1}^{N_{\text {el }}} \iint_{\Omega^{e}}\left[Q_{11}\left(\frac{\partial \widetilde{u}^{e}}{\partial x}\right)^{2}+2 Q_{12}\left(\frac{\partial \widetilde{v}^{e}}{\partial y}\right)\left(\frac{\partial \widetilde{u}^{e}}{\partial x}\right)+Q_{22}\left(\frac{\partial \widetilde{v}^{e}}{\partial y}\right)^{2}+Q_{66}\left(\frac{\partial \widetilde{u}^{e}}{\partial y}+\frac{\partial \widetilde{v}^{e}}{\partial x}\right)^{2}-\omega_{l}^{2} \rho\left(\left(\widetilde{u}^{e}\right)^{2}+\left(\widetilde{v}^{e}\right)\right)^{2}\right] d x d y\right\} \\
& =0
\end{aligned}
$$




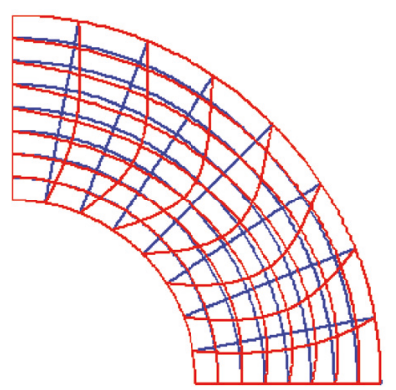

$\Omega^{1}=3.1710$

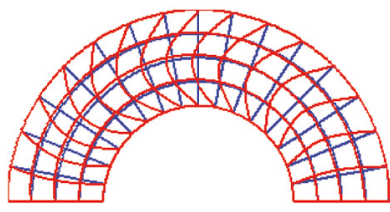

$\Omega^{1}=2.7845$

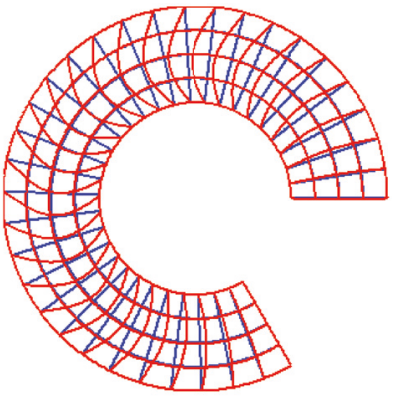

$\Omega^{1}=2.6964$

(a)

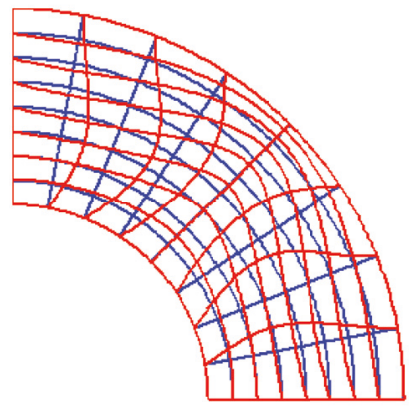

$\Omega^{2}=4.2199$

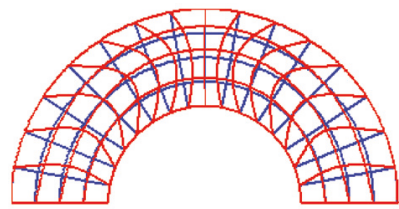

$\Omega^{2}=3.1545$

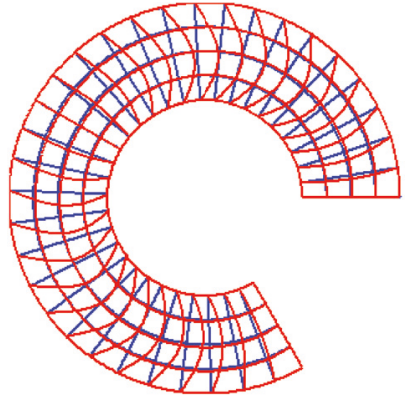

$\Omega^{2}=2.8404$

(b)
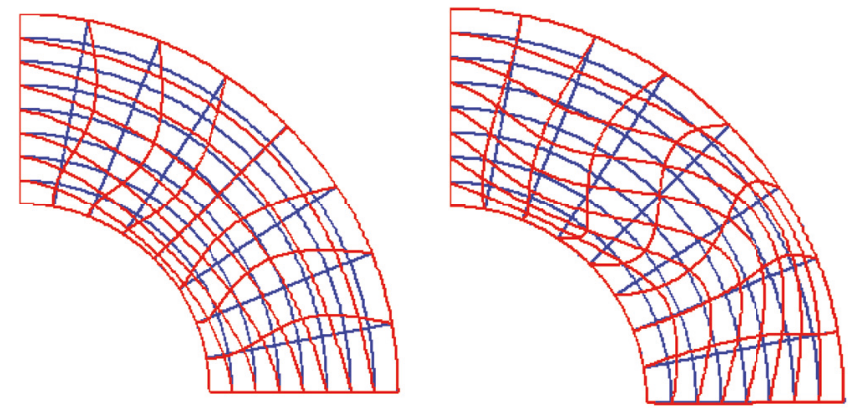

$\Omega^{3}=4.5769$

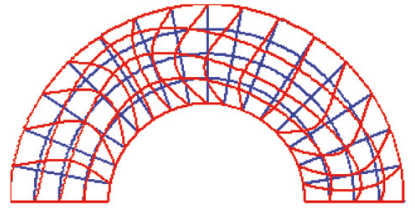

$\Omega^{3}=3.6574$

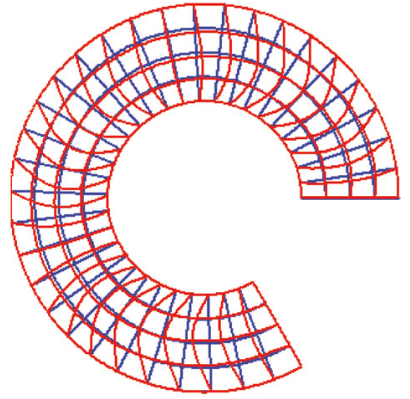

$\Omega^{3}=3.0612$

(c)

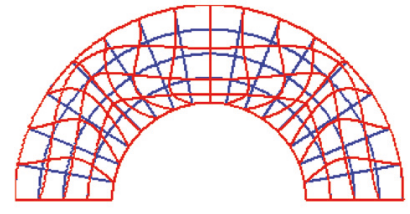

$\Omega^{4}=4.1550$

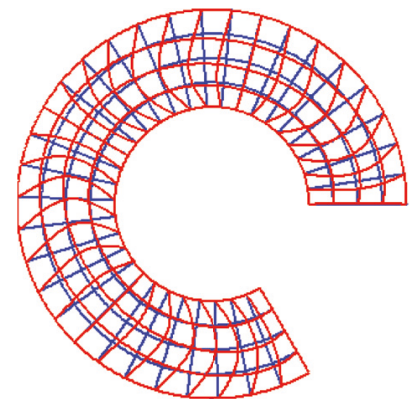

$\Omega^{4}=3.3369$

(d)

FIGURE 5: The first four mode shapes of CCCC annular sector plate $\left(R_{0} / R_{1}=0.5\right)$ with different sector angles, that is, $\phi=90^{\circ}, 180^{\circ}$, and $300^{\circ}$.

Therefore, the in-plane vibration governing expression can be written as

$$
\left[\mathbf{K}-\omega_{l}^{2} \mathbf{M}\right]\left\{\widetilde{\boldsymbol{\varphi}}_{l}\right\}=0,
$$

where $\mathbf{M}$ and $\mathbf{K}$ are, respectively, the global mass and stiffness matrices for sector plate. $\omega_{l}$ denotes the $l$ th natural frequency of sector plate, and $\left\{\widetilde{\boldsymbol{\varphi}}_{l}\right\}=\{\widetilde{\mathbf{u}}, \widetilde{\mathbf{v}}\}$ denotes the corresponding eigenvector nodal displacements, in which displacement component vectors $\widetilde{\mathbf{u}}$ and $\widetilde{\mathbf{v}}$ are written as

$$
\begin{aligned}
& \widetilde{\mathbf{u}}=\left\{\widetilde{u}_{1}, \widetilde{u}_{2}, \widetilde{u}_{3} \cdots \widetilde{u}_{n \cdot m}\right\}^{\mathrm{T}} \\
& \widetilde{\mathbf{v}}=\left\{\widetilde{v}_{1}, \widetilde{v}_{2}, \widetilde{v}_{3} \cdots \widetilde{v}_{n \cdot m}\right\}^{\mathrm{T}} .
\end{aligned}
$$

The eigenproblem in (30) can be solved by using Arnoldi Method [51, 52], and the mode shapes can be expressed by multiplying the NURBS basis functions with eigenvector.
To calculate the global stiffness matrix and mass matrix in (30), each local element mass matrix and stiffness matrix should be computed according to (29). Therefore, the local element stiffness matrix can be written as

$$
\mathbf{K}^{\mathbf{e}}=\left[\begin{array}{ll}
\mathbf{K}^{11} & \mathbf{K}^{12} \\
\mathbf{K}^{21} & \mathbf{K}^{22}
\end{array}\right] .
$$

The explicit forms of the local element stiffness matrix $\mathbf{K}^{\mathbf{e}}$ are given by

$$
\begin{aligned}
& K_{i j}^{11}=\iint_{\Omega^{e}}\left(Q_{11} \frac{\partial R_{i}}{\partial x} \frac{\partial R_{j}}{\partial x}+Q_{66} \frac{\partial R_{i}}{\partial y} \frac{\partial R_{j}}{\partial y}\right) d x d y \\
& K_{i j}^{12}=\iint_{\Omega^{e}}\left(Q_{12} \frac{\partial R_{i}}{\partial x} \frac{\partial R_{j}}{\partial y}+Q_{66} \frac{\partial R_{i}}{\partial y} \frac{\partial R_{j}}{\partial x}\right) d x d y
\end{aligned}
$$




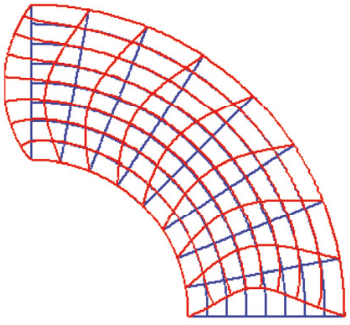

$\Omega^{1}=2.5350$

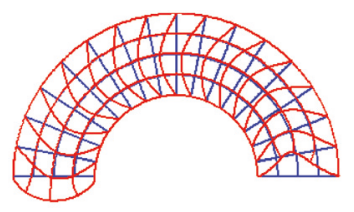

$\Omega^{1}=2.5823$

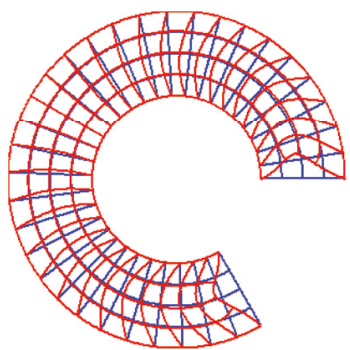

$\Omega^{1}=2.5989$

(a)

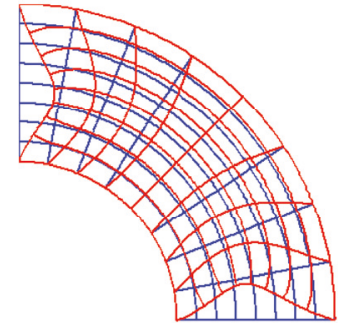

$\Omega^{2}=2.9788$

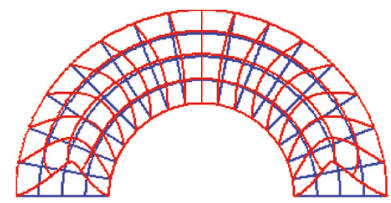

$\Omega^{2}=2.6746$

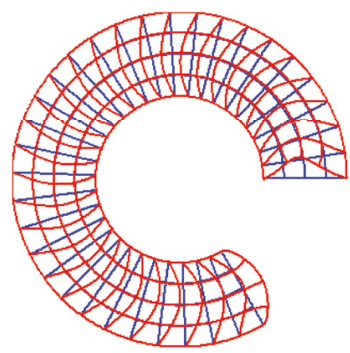

$\Omega^{2}=2.6212$

(b)

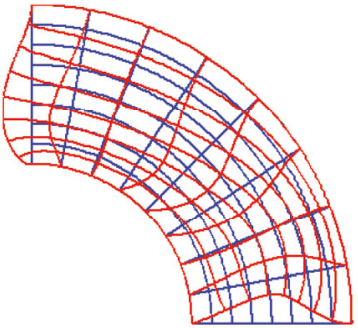

$\Omega^{3}=3.9951$

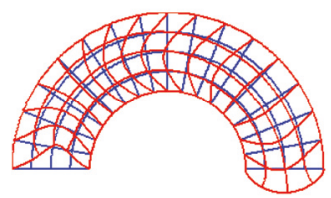

$\Omega^{3}=3.0658$

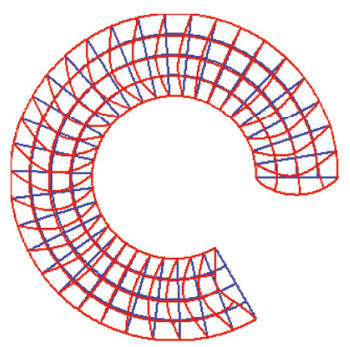

$\Omega^{3}=2.7858$

(c)

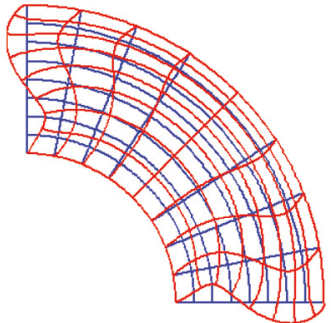

$\Omega^{4}=4.3373$

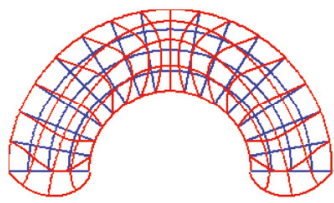

$\Omega^{4}=3.5691$

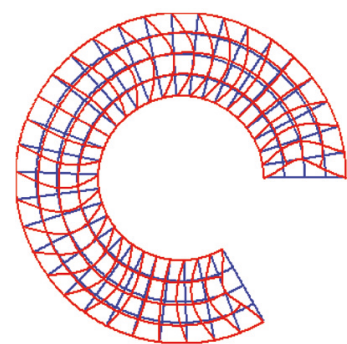

$\Omega^{4}=3.0088$

(d)

Figure 6: The first four mode shapes of CFCF annular sector plate $\left(R_{0} / R_{1}=0.5\right)$ with different sector angles, that is, $\phi=90^{\circ}, 180^{\circ}$, and $300^{\circ}$.

$$
\begin{aligned}
& K_{i j}^{21}=\iint_{\Omega^{e}}\left(Q_{12} \frac{\partial R_{i}}{\partial y} \frac{\partial R_{j}}{\partial x}+Q_{66} \frac{\partial R_{i}}{\partial x} \frac{\partial R_{j}}{\partial y}\right) d x d y \\
& K_{i j}^{22}=\iint_{\Omega^{e}}\left(Q_{22} \frac{\partial R_{i}}{\partial y} \frac{\partial R_{j}}{\partial y}+Q_{66} \frac{\partial R_{i}}{\partial x} \frac{\partial R_{j}}{\partial x}\right) d x d y .
\end{aligned}
$$

Each local element mass matrix for sector plates can be written as

$$
\mathbf{M}^{\mathbf{e}}=\left[\begin{array}{ll}
\mathbf{M}^{11} & \mathbf{M}^{12} \\
\mathbf{M}^{21} & \mathbf{M}^{22}
\end{array}\right]
$$

The explicit forms of the local element mass matrix $\mathbf{M}^{\mathbf{e}}$ are given by

$$
\begin{aligned}
& M_{i j}^{11}=\iint_{\Omega^{e}}\left(\rho R_{i} R_{j}\right) d x d y \\
& M_{i j}^{12}=M_{i j}^{21}=0 \\
& M_{i j}^{22}=\iint_{\Omega^{e}}\left(\rho R_{i} R_{j}\right) d x d y .
\end{aligned}
$$

By using integral transform which is determined in (23), the integral region of element can be transformed to numerical integral region. Then numerical integration can be obtained by applying the standard Gauss-Legendre quadrature.
The global stiffness and mass matrices of annular sector plate can be obtained by using (29)-(35). For the stiffness and mass matrices of the annular plate, circular sector plate, and circular plate, a coupling coincident control points scheme mentioned above is used. When the angle $\phi$ of the annular sector plate is equal to $2 \pi$ and with coupling the edges $\phi=0$, $\phi=2 \pi$, the annular sector plate can be regarded as an annular plate. When inner radius $R_{0}$ of the annular sector plate is zero and with coupling the control points in the inner radius $R_{0}$, the annular sector plate can be regarded as a circular sector plate. In a similar way, circular plate can be obtained when the angle $\phi$ of the circular sector plate is equal to $2 \pi$ and with coupling the edges $\phi=0, \phi=2 \pi$.

2.5. Refinement Algorithms. The accuracy of FEM computational solutions is greatly related to the number of elements and the order of functions used to describe unknown field variables. To capture fast convergence in the solution field, it is essential that the discretization of intricate geometries can be successively refined. The basic B-spline refinement mechanisms are different from the counterparts of the FEM. One of the main advantages of a discretization tool based on the B-spline basis functions is the ability to apply different refinements algorithms in a simple manner. Three types of refinement algorithms [53] are generally used in the IGA. 


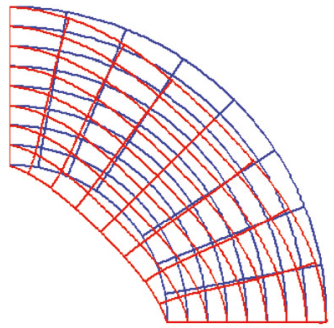

$\Omega^{1}=1.1492$

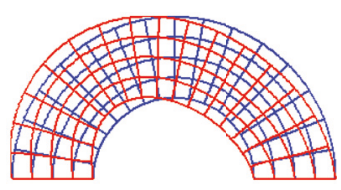

$\Omega^{1}=0.4655$

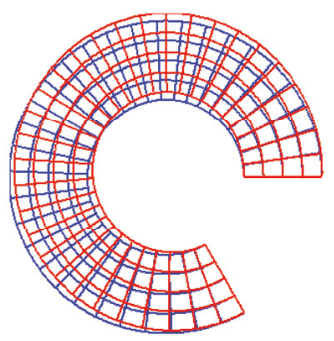

$\Omega^{1}=0.1427$

(a)

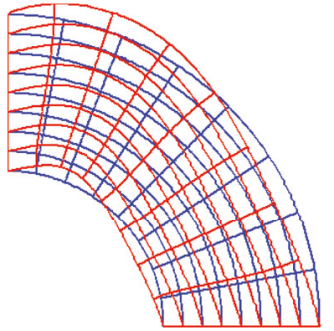

$\Omega^{2}=1.4149$

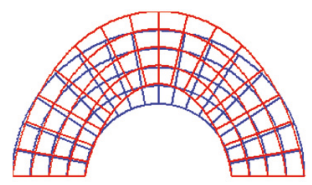

$\Omega^{2}=0.7004$

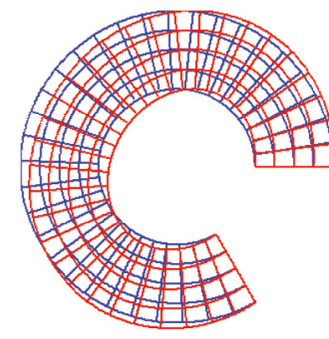

$\Omega^{2}=0.3280$

(b)

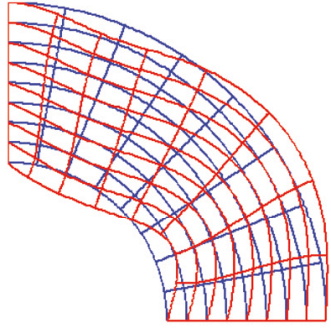

$\Omega^{3}=1.9391$

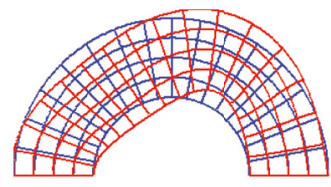

$\Omega^{3}=1.0857$

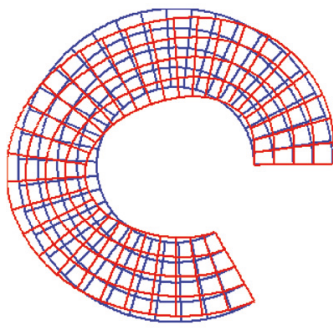

$\Omega^{3}=0.5741$

(c)

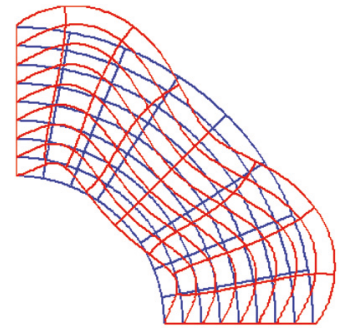

$\Omega^{4}=2.6627$

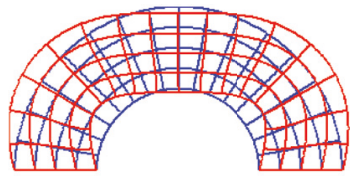

$\Omega^{4}=1.1836$

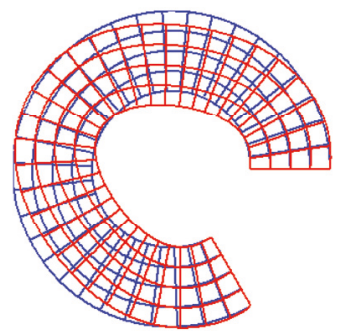

$\Omega^{4}=0.7826$

(d)

Figure 7: The first four mode shapes of FCFC annular sector plate $\left(R_{0} / R_{1}=0.5\right)$ with different sector angles, that is, $\phi=90^{\circ}, 180^{\circ}$, and $300^{\circ}$.

In the IGA, knot insertion is a strategy that inserts additional knots into the knot vectors. In this subdivision strategy, both the number and control points of element are changed but the order of basis functions is maintained. Degree elevation is a strategy that increases the order of B-spline basis functions. In this strategy, both the order and control points of basis B-spline functions are changed yet knot span is not increased. The knot insertion and degree elevation, respectively, are analogous to the $h$ - and $p$ refinement of the standard FEA. In addition, the combination of knot insertion with degree elevation is the ability to refine discretization. A strategy that first elevates the order of NURBS basis functions and then inserted a unique knot into a nonzero knot span is said to be $k$-refinement. As an efficient and robust scheme, this strategy can increase the number of elements and provide higher order of basis functions.

\section{Numerical Results and Discussions}

In this part, the convergence of the current method is first tested. Then, several selected numerical examples of the inplane vibration analysis for sector, annular, and circular plates are given to verify the validity and accuracy of this IGA method. Free (F) and clamped (C) boundary conditions are considered. For instance, CCCC and FFFF, respectively, represent all clamped and free sides, CCCF means the fourth side is free and other three sides are fixed. The sequence of boundary edges for the sector plates is defined in Figure 3. Then, some free in-plane vibration mode shapes of sector plates are plotted and relevant frequencies are presented. Finally, the effects of some parameters study examples are also given. In the following analysis, the isotropic annular, circular, and sector plates are considered as thin plates. The nondimensional frequency parameter $\left.\Omega=\left(2 \omega R_{1} / \pi\right) \sqrt{\left(\rho /\left(E\left(1-\nu^{2}\right)\right)\right.}\right)$ is used if no special statement is given; the material parameters of the different types of annular sector, annular, circular sector, and circular plates are $E=70 \mathrm{Gpa}, \nu=0.3$, and $\rho=2700 \mathrm{~kg} / \mathrm{m}^{3}$.

3.1. Convergence and Validation. The annular sector plate as an example is applied to test the convergence of the present approach for free in-plane natural frequencies. For conciseness and convenience, three refinement schemes such as $h$ , $p$-, and $k$-refinements which elevate the polynomial order and insert knots with preserving the geometry and parameterization are employed. Then, the first eight nondimensional frequency parameters of the CCCC annular sector plate by using different orders and elements are shown in Table 1. The plates are characterized by the following geometry constants: 
TABLE 1: Convergence of frequency parameters $\Omega=\left(2 \omega R_{1} / \pi\right) \sqrt{\left(\rho /\left(E\left(1-v^{2}\right)\right)\right.}$ $\Omega$ of plates with CCCC boundaries and inner-to-outer radii ratios: $R_{0} / R_{1}=0.5$.

\begin{tabular}{|c|c|c|c|c|c|c|c|c|c|}
\hline \multirow{2}{*}{ Order } & \multirow{2}{*}{ Element } & \multicolumn{8}{|c|}{ Mode number } \\
\hline & & 1 & 2 & 3 & 4 & 5 & 6 & 7 & 8 \\
\hline \multirow{3}{*}{$p=q=2$} & $8 \times 8$ & 4.4070 & 4.8359 & 5.5223 & 6.9141 & 7.0940 & 7.3009 & 8.0867 & 8.7015 \\
\hline & $16 \times 16$ & 4.4065 & 4.8353 & 5.5204 & 6.9101 & 7.0884 & 7.2908 & 8.0748 & 8.6908 \\
\hline & $32 \times 32$ & 4.4064 & 4.8352 & 5.5203 & 6.9098 & 7.0880 & 7.2902 & 8.0742 & 8.6901 \\
\hline \multirow{3}{*}{$p=q=3$} & $8 \times 8$ & 4.4066 & 4.8355 & 5.5204 & 6.9101 & 7.0883 & 7.2908 & 8.0748 & 8.6908 \\
\hline & $16 \times 16$ & 4.4065 & 4.8353 & 5.5203 & 6.9099 & 7.0881 & 7.2903 & 8.0742 & 8.6902 \\
\hline & $32 \times 32$ & 4.4064 & 4.8352 & 5.5203 & 6.9098 & 7.0880 & 7.2902 & 8.0741 & 8.6901 \\
\hline \multirow{3}{*}{$p=q=4$} & $8 \times 8$ & 4.4066 & 4.8355 & 5.5204 & 6.9099 & 7.0882 & 7.2905 & 8.0744 & 8.6903 \\
\hline & $16 \times 16$ & 4.4065 & 4.8354 & 5.5204 & 6.9100 & 7.0881 & 7.2903 & 8.0743 & 8.6903 \\
\hline & $32 \times 32$ & 4.4065 & 4.8353 & 5.5203 & 6.9099 & 7.0880 & 7.2902 & 8.0742 & 8.6902 \\
\hline \multirow{3}{*}{$p=q=5$} & $8 \times 8$ & 4.4067 & 4.8356 & 5.5205 & 6.9099 & 7.0882 & 7.2905 & 8.0744 & 8.6903 \\
\hline & $16 \times 16$ & 4.4066 & 4.8354 & 5.5204 & 6.9100 & 7.0882 & 7.2904 & 8.0743 & 8.6903 \\
\hline & $32 \times 32$ & 4.4065 & 4.8353 & 5.5203 & 6.9099 & 7.0881 & 7.2903 & 8.0742 & 8.6902 \\
\hline
\end{tabular}

TABLE 2: Comparison of frequency parameters $\Omega=\omega R_{1} \sqrt{\rho / E}$ for annular sector plates with various boundaries and inner-to-outer radii ratios: $R_{0} / R_{1}=0.5$.

\begin{tabular}{cccccccccccc}
\hline \multirow{2}{*}{$\phi\left(^{\circ}\right)$} & Mode & \multicolumn{3}{c}{ CCCC } & & & \multicolumn{2}{c}{ CFCF } & \multicolumn{3}{c}{ FFFF } \\
& & Ref. [23] & Ref. [25] & Present & Ref. [23] & Ref. [25] & Present & Ref. [25] & Present & Ref. [25] & Present \\
\hline & 1 & 8.1592 & 8.1500 & 8.1527 & 3.4489 & 3.4788 & 3.4774 & 4.8589 & 4.8777 & 5.3157 & 5.3112 \\
& 2 & 8.8379 & 8.8085 & 8.8122 & 6.2770 & 6.2179 & 6.2184 & 5.6760 & 5.6938 & 6.8500 & 6.8399 \\
30 & 3 & 10.1089 & 10.2116 & 10.2138 & 6.4037 & 6.4445 & 6.4441 & 5.7026 & 5.7199 & 7.4560 & 7.5122 \\
& 4 & 12.7648 & 12.7775 & 12.7822 & 8.3676 & 8.3194 & 8.3189 & 6.1162 & 6.1370 & 10.2296 & 10.2655 \\
& 5 & 13.2623 & 13.3090 & 13.3143 & 8.3358 & 8.4499 & 8.4478 & 7.6716 & 7.6990 & 10.7897 & 10.8673 \\
& 6 & 13.2555 & 13.3195 & 13.3225 & 9.4104 & 9.4264 & 9.4264 & 8.5692 & 8.6995 & 11.5209 & 11.5250 \\
\hline & 1 & 4.7576 & 4.7508 & 4.7516 & 3.7834 & 3.8017 & 3.7986 & 1.5633 & 1.5641 & 4.0055 & 4.0121 \\
& 2 & 6.3458 & 6.3216 & 6.3233 & 4.4797 & 4.4676 & 4.4636 & 2.6785 & 2.6702 & 5.4346 & 5.3987 \\
90 & 3 & 6.9512 & 6.8564 & 6.8582 & 6.0711 & 5.9866 & 5.9864 & 2.9795 & 2.9792 & 6.5046 & 6.4447 \\
& 4 & 6.9793 & 6.8921 & 6.8937 & 6.5393 & 6.5032 & 6.4992 & 4.4513 & 4.4293 & 6.6321 & 6.5915 \\
& 5 & 7.5749 & 7.5573 & 7.5588 & 6.5952 & 6.5497 & 6.5504 & 4.4627 & 4.4754 & 7.0237 & 6.9826 \\
& 6 & 8.0831 & 8.0973 & 8.0983 & 6.7786 & 6.7742 & 6.7739 & 4.6668 & 4.6575 & 7.3563 & 7.3541 \\
\hline
\end{tabular}

$R_{1}=1 \mathrm{~m}, R_{0} / R_{1}=0.5$, and $\phi=\pi / 4$. Obviously, the computed results in Table 1 show a great convergence trend.

The comparisons of frequency parameters of these plates including annular sector plate, annular plate, and circular sector plate are shown in Tables 2-4. The first six dimensionless natural frequencies $\Omega=\omega R_{1} \sqrt{\rho / E}$ of annular sector plates are presented Table 2 . In this table, the inner-to-outer radii ratios $R_{0} / R_{1}=0.5$, the sector angles $\phi=30^{\circ}, 90^{\circ}$, and different boundary conditions such as CCCC, CFCF, FFFF, and CCCF are taken into account. Some results in available literature reported by Singh and Muhammad [23] using traditional FEM and Shi et al. [25] using Ritz method are used in Table 2 to validate the accuracy of results of annular sector plate with the present method. From Table 2, a good agreement can be obtained by observing these results. The discrepancies of the results can be negligible.
Besides, annular and circular sector plates under this investigation are considered as special cases on the basis of the model of annular sector plate. As mentioned above, they are obtained by using a scheme for coupling coincident control points. Therefore, the results of frequency parameters of these plates should be investigated. The nondimensional frequency parameters results of the annular plate are presented in Table 3. The inner-to-outer radii ratios used for this table are 0.2 and 0.4. Different boundary conditions FF, FC, and CC are taken into consideration. Similarly, the natural frequencies of annular plate are compared with the results which are taken from Irie et al. [19] using transfer matrix method and Bashmal et al. [20] using Rayleigh-Ritz method. Table 4 presents the first nondimensional nine frequency parameters of the circular sector plate. The sector angles $\phi=30^{\circ}, 90^{\circ}$ and boundary conditions CCC, CFC, and FCF 
TABLE 3: Comparison of frequency parameters $\Omega=\left(2 \omega R_{1} / \pi\right) \sqrt{\left(\rho /\left(E\left(1-\nu^{2}\right)\right)\right)}$ for annular plates with various boundary conditions.

\begin{tabular}{|c|c|c|c|c|c|c|c|c|c|c|}
\hline \multirow{2}{*}{$R_{0} / R_{1}$} & \multirow{2}{*}{ Mode } & \multicolumn{3}{|c|}{$\mathrm{FF}$} & \multicolumn{3}{|c|}{ FC } & \multicolumn{3}{|c|}{$\mathrm{CC}$} \\
\hline & & Ref. [19] & Ref. [20] & Present & Ref. [19] & Ref. [20] & Present & Ref. [19] & Ref. [20] & Present \\
\hline \multirow{8}{*}{0.2} & \multirow{2}{*}{$n=1$} & 1.156 & 1.155 & 1.156 & 1.472 & 1.473 & 1.472 & 1.947 & 1.963 & 1.947 \\
\hline & & 2.688 & 2.687 & 2.680 & 2.311 & 2.313 & 2.310 & 2.840 & 2.870 & 2.840 \\
\hline & \multirow{2}{*}{$n=2$} & 0.777 & 0.777 & 0.777 & 1.786 & 1.788 & 1.472 & 2.363 & 2.374 & 2.363 \\
\hline & & 1.681 & 1.680 & 1.683 & 2.762 & 2.765 & 2.762 & 3.050 & 3.066 & 3.050 \\
\hline & \multirow{2}{*}{$n=3$} & 1.449 & 1.450 & 1.449 & 2.580 & 2.584 & 2.580 & 2.845 & 2.857 & 2.844 \\
\hline & & 2.379 & 2.379 & 2.380 & 3.399 & 3.364 & 3.399 & 3.571 & 3.582 & 3.570 \\
\hline & \multirow{2}{*}{$n=4$} & 1.936 & 1.935 & 1.936 & 3.296 & 3.301 & 3.296 & 3.359 & 3.366 & 3.358 \\
\hline & & 3.070 & 3.069 & 3.067 & 4.123 & 4.130 & 4.123 & 4.200 & 4.051 & 4.199 \\
\hline \multirow{8}{*}{0.4} & \multirow{2}{*}{$n=1$} & 1.177 & 1.177 & 1.177 & 1.761 & 1.764 & 1.761 & 2.399 & 2.418 & 2.399 \\
\hline & & 2.829 & 2.829 & 2.828 & 2.454 & 2.458 & 2.454 & 3.712 & 3.743 & 3.713 \\
\hline & \multirow{2}{*}{$n=2$} & 0.504 & 0.504 & 0.505 & 1.904 & 1.913 & 1.903 & 2.814 & 2.831 & 2.814 \\
\hline & & 1.715 & 1.714 & 1.714 & 2.901 & 2.905 & 2.901 & 3.715 & 3.741 & 3.716 \\
\hline & \multirow{2}{*}{$n=3$} & 1.132 & 1.133 & 1.132 & 2.248 & 2.252 & 2.249 & 3.293 & 3.314 & 3.293 \\
\hline & & 2.341 & 2.340 & 2.272 & 3.497 & 3.501 & 3.496 & 3.931 & 3.953 & 3.931 \\
\hline & \multirow{2}{*}{$n=4$} & 1.736 & 1.736 & 1.736 & 2.767 & 2.770 & 2.767 & 3.699 & 3.750 & 3.699 \\
\hline & & 2.957 & 2.956 & 2.953 & 4.109 & 4.115 & 4.109 & 4.400 & 4.416 & 4.399 \\
\hline
\end{tabular}

TABLE 4: Comparison of the first nine frequency parameters $\Omega=\left(2 \omega R_{1} / \pi\right) \sqrt{\left(\rho /\left(E\left(1-\nu^{2}\right)\right)\right)}$ for circular sector plate with various boundary conditions.

\begin{tabular}{|c|c|c|c|c|c|c|c|c|c|c|c|}
\hline \multirow{2}{*}{$\mathrm{BC}$} & \multirow{2}{*}{$\phi\left({ }^{\circ}\right)$} & \multirow{2}{*}{ Method } & \multicolumn{9}{|c|}{ Mode sequence number } \\
\hline & & & 1 & 2 & 3 & 4 & 5 & 6 & 7 & 8 & 9 \\
\hline \multirow{8}{*}{ CCC } & \multirow{2}{*}{60} & ANSYS & 3.4710 & 3.5239 & 4.2842 & 5.3930 & 5.5591 & 5.8322 & 5.8813 & 5.9433 & 7.0236 \\
\hline & & Present & 3.4716 & 3.5246 & 4.2847 & 5.3941 & 5.5601 & 5.8331 & 5.8821 & 5.9443 & 7.0243 \\
\hline & \multirow{2}{*}{90} & ANSYS & 2.7035 & 2.9895 & 3.4421 & 4.1459 & 4.5198 & 4.5668 & 4.9401 & 5.1551 & 5.4300 \\
\hline & & Present & 2.7039 & 2.9900 & 3.4423 & 4.1466 & 4.5202 & 4.5676 & 4.9407 & 5.1560 & 5.4306 \\
\hline & \multirow{2}{*}{180} & ANSYS & 1.8735 & 2.3630 & 2.5835 & 2.8205 & 3.1101 & 3.1957 & 3.6007 & 3.7763 & 3.8302 \\
\hline & & Present & 1.8737 & 2.3632 & 2.5836 & 2.8207 & 3.1103 & 3.1961 & 3.6010 & 3.7768 & 3.8305 \\
\hline & \multirow{2}{*}{270} & ANSYS & 1.6512 & 1.9271 & 2.2385 & 2.5417 & 2.6073 & 2.6320 & 2.9348 & 3.0282 & 3.2372 \\
\hline & & Present & 1.6512 & 1.9274 & 2.2387 & 2.5419 & 2.6074 & 2.6324 & 2.9351 & 3.0285 & 3.2375 \\
\hline \multirow{8}{*}{$\mathrm{CFC}$} & \multirow{2}{*}{60} & ANSYS & 2.0725 & 2.3418 & 2.9577 & 3.9833 & 4.4296 & 4.4502 & 4.7109 & 4.9565 & 5.2712 \\
\hline & & Present & 2.0727 & 2.3420 & 2.9579 & 3.9835 & 4.4303 & 4.4507 & 4.7113 & 4.9568 & 5.2713 \\
\hline & \multirow{2}{*}{90} & ANSYS & 1.5530 & 1.6721 & 2.1934 & 2.7998 & 3.2069 & 3.4231 & 3.8270 & 4.0418 & 4.0815 \\
\hline & & Present & 1.5532 & 1.6723 & 2.1934 & 2.8000 & 3.2073 & 3.4234 & 3.8274 & 4.0421 & 4.0817 \\
\hline & \multirow{2}{*}{180} & ANSYS & 0.7003 & 1.1850 & 1.4080 & 1.6112 & 1.8608 & 1.9677 & 2.4200 & 2.4398 & 2.6848 \\
\hline & & Present & 0.7003 & 1.1851 & 1.4081 & 1.6112 & 1.8609 & 1.9677 & 2.4201 & 2.4400 & 2.6849 \\
\hline & \multirow{2}{*}{270} & ANSYS & 0.3983 & 0.7898 & 1.0803 & 1.3769 & 1.4061 & 1.4591 & 1.7509 & 1.7527 & 2.0124 \\
\hline & & Present & 0.3983 & 0.7898 & 1.0803 & 1.3770 & 1.4061 & 1.4592 & 1.7510 & 1.7527 & 2.0125 \\
\hline \multirow{8}{*}{ FCF } & \multirow{2}{*}{60} & ANSYS & 0.8416 & 1.5705 & 1.6204 & 2.6790 & 3.1841 & 3.5447 & 3.7060 & 3.8662 & 4.0591 \\
\hline & & Present & 0.8417 & 1.5707 & 1.6204 & 2.6791 & 3.1844 & 3.5449 & 3.7061 & 3.8663 & 4.0594 \\
\hline & \multirow{2}{*}{90} & ANSYS & 1.0527 & 1.5126 & 1.7130 & 2.5865 & 2.8986 & 3.1485 & 3.1677 & 3.6040 & 4.0099 \\
\hline & & Present & 1.0528 & 1.5127 & 1.7131 & 2.5867 & 2.8987 & 3.1486 & 3.1679 & 3.6041 & 4.0100 \\
\hline & \multirow{2}{*}{180} & ANSYS & 1.3000 & 1.3378 & 1.6175 & 2.0817 & 2.3425 & 2.4921 & 2.6675 & 3.1048 & 3.1080 \\
\hline & & Present & 1.3001 & 1.3379 & 1.6176 & 2.0818 & 2.3427 & 2.4922 & 2.6676 & 3.1050 & 3.1081 \\
\hline & \multirow{2}{*}{270} & ANSYS & 1.2704 & 1.4099 & 1.4850 & 1.7801 & 2.1763 & 2.2147 & 2.2991 & 2.5909 & 2.7198 \\
\hline & & Present & 1.2705 & 1.4099 & 1.4850 & 1.7799 & 2.1765 & 2.2146 & 2.2992 & 2.5910 & 2.7200 \\
\hline
\end{tabular}




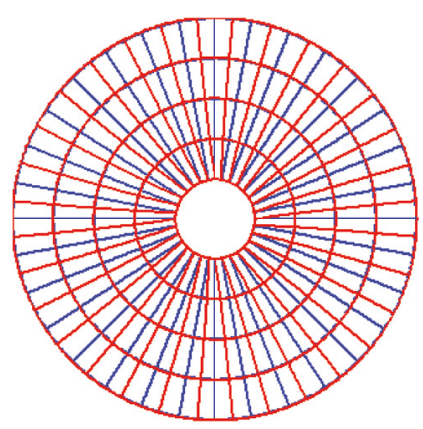

$\Omega^{1}=0.2466$

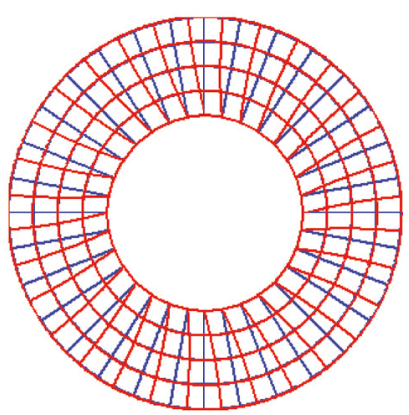

$\Omega^{1}=0.8167$

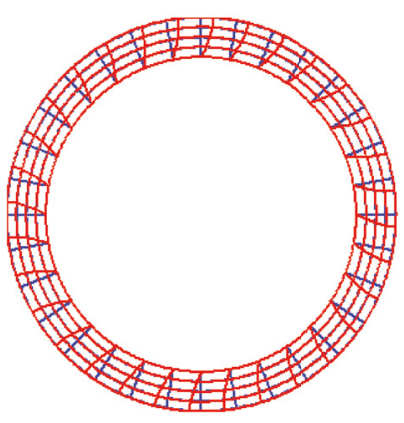

$\Omega^{1}=2.8252$

(a)

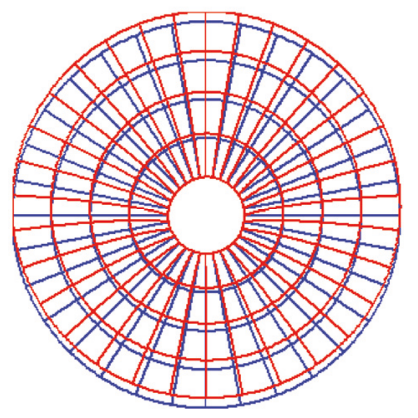

$\Omega^{2}=\Omega^{3}=0.6432$

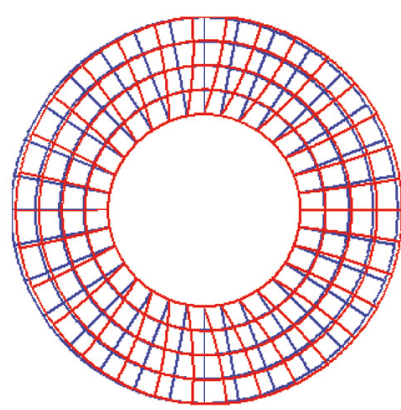

$\Omega^{2}=\Omega^{3}=1.0807$

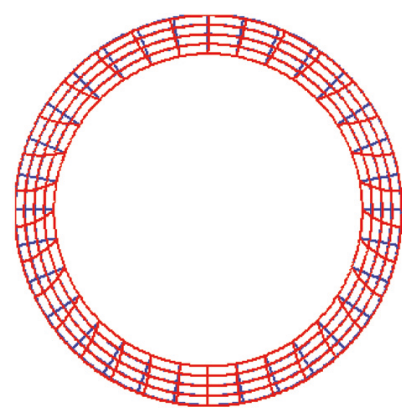

$\Omega^{2}=\Omega^{3}=2.9115$

(b)

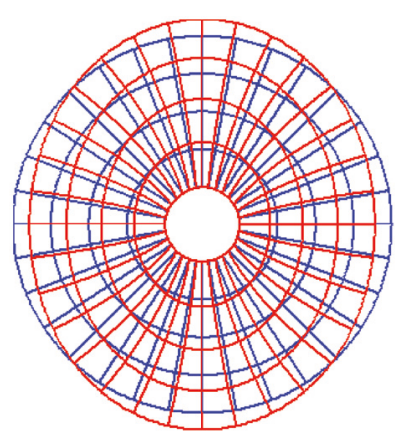

$\Omega^{4}=\Omega^{5}=1.0784$

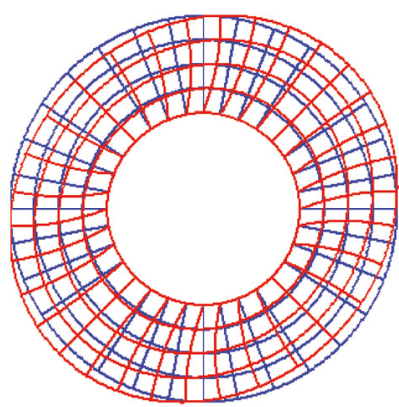

$\Omega^{4}=\Omega^{5}=1.5753$

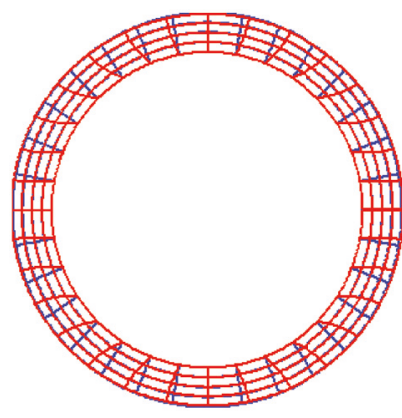

$\Omega^{4}=\Omega^{5}=3.1557$

(c)

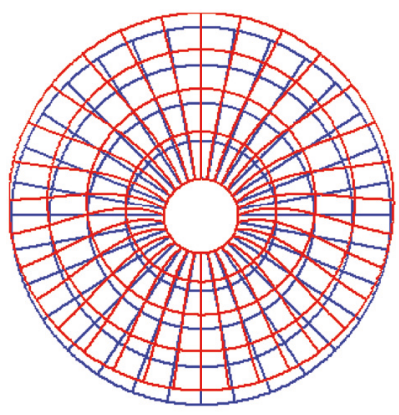

$\Omega^{6}=\Omega^{7}=1.4838$

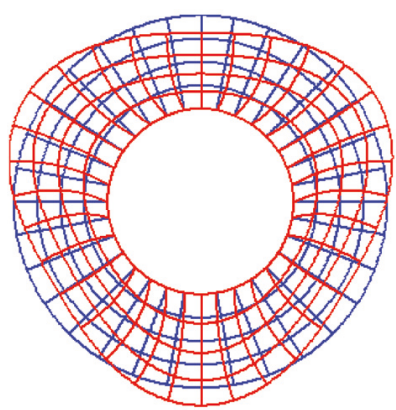

$\Omega^{6}=\Omega^{7}=1.9553$

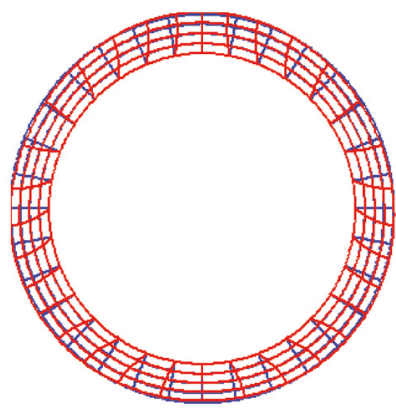

$\Omega^{6}=\Omega^{7}=3.5234$

(d)

FIgURE 8: The first seven mode shapes of CF annular plate with different inner-to-outer radii ratios, that is, $R_{0} / R_{1}=0.2,0.5$, and 0.8 .

are taken into consideration. To validate these results of the present method in Table 4, the natural frequency parameters computed by ANSYS based on the FEM are utilized. In this ANSYS computation, the type and the size of element are, respectively, shell 82 and 0.02 , and mapped meshing grid is adapted. Based on the above description of tables, it is obvious that this IGA method has a great accuracy and efficiency to evaluate the natural in-plane vibration frequencies for the annular sector, annular, and circular sector plates.

Figures 5-7 depict first four mode shapes and relevant frequency parameters of the free in-plane vibration for annular sector plate with different geometric parameters and boundary conditions. From these figures, it can be easily seen that in-plane vibration mode shapes are more complicated than their flexural vibration modes even though they belong to lower-order modes. For example, the deformation of extension and compression in one region can suddenly change into a shear state in another region as mode varies. One of the reasons is maybe that the in-plane vibration frequency is determined by many factors rather than individual variable of the plate. The deformation of the first mode extends and compresses in the circumferential direction under CCCC and CFCF boundary conditions but in the radial direction under FCFC boundary condition. The variations of mode shapes with sector angle also are obviously seen. Therefore, the boundary conditions and the geometry parameters play significant roles in their in-plane vibration mode shapes. Figures 8-10 present some mode shapes, corresponding to 


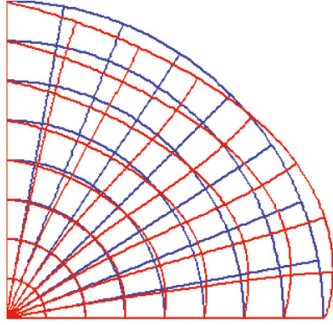

$\Omega^{1}=1.5531$

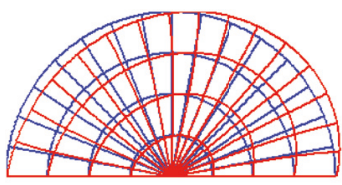

$\Omega^{1}=0.7003$

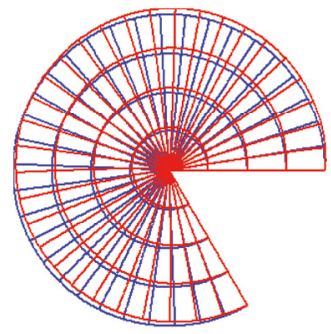

$\Omega^{1}=0.3413$

(a)

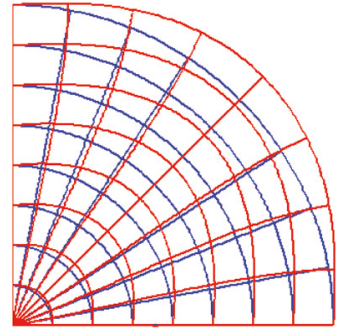

$\Omega^{2}=1.6723$

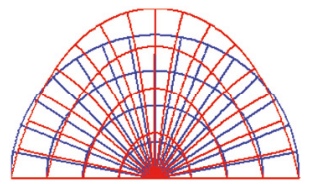

$\Omega^{2}=1.1851$

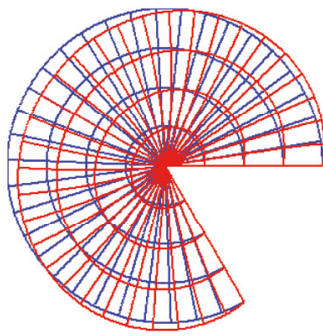

$\Omega^{2}=0.6904$

(b)

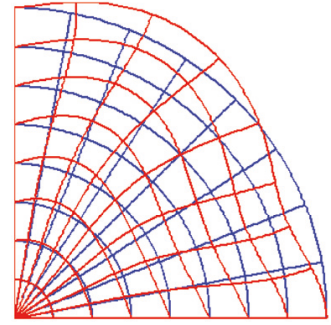

$\Omega^{3}=2.1935$

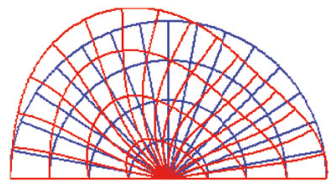

$\Omega^{3}=1.4081$

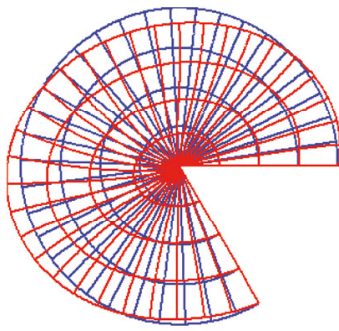

$\Omega^{3}=0.9857$

(c)

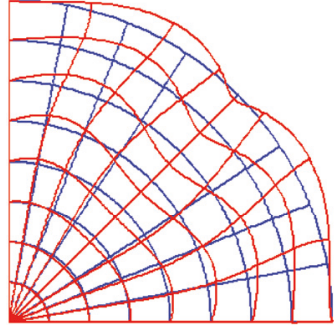

$\Omega^{4}=2.7999$

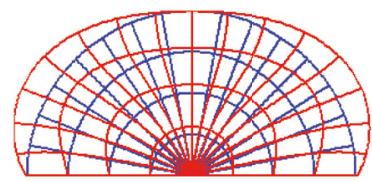

$\Omega^{4}=1.6112$

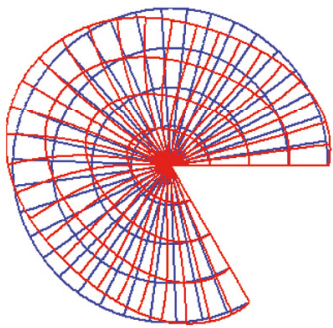

$\Omega^{4}=1.2652$

(d)

FIgURE 9: The first seven mode shapes of CFC circular sector plate with different sector angles, that is, $\phi=90^{\circ}, 180^{\circ}$, and $300^{\circ}$.

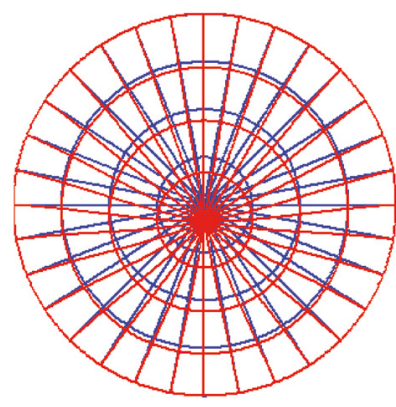

(a) $\Omega^{1}=1.3692$

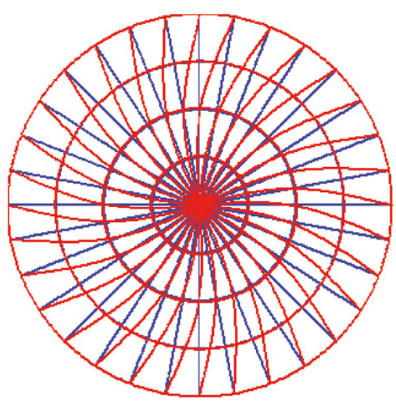

(b) $\Omega^{2}=\Omega^{3}=1.5859$

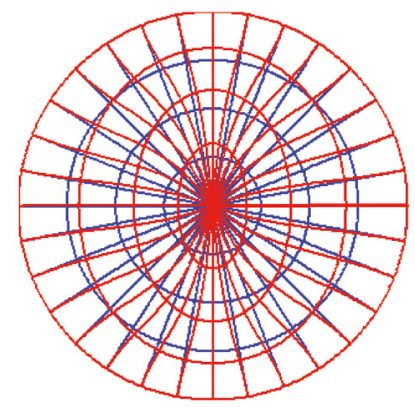

(c) $\Omega^{4}=\Omega^{5}=2.1321$

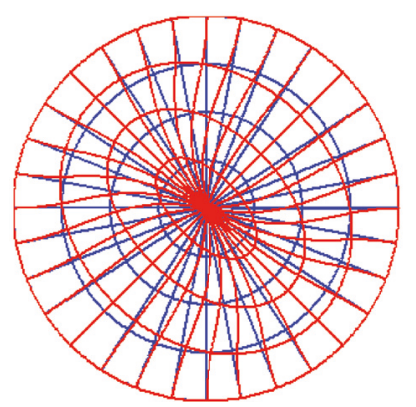

(d) $\Omega^{6}=\Omega^{7}=2.2237$

FIGURE 10: The first four mode shapes of C circular plate with different boundary conditions.

frequency parameters of annular, circular, and circular plates with different geometric parameters, respectively.

3.2. The Effects of Parameters. In numerical studies, the effects of several key parameters are investigated in Figures 11-13. The order $p=q=3$ and the element $32 \times 32$ are used to calculate the frequency parameters. The change trends of the first three frequencies for annular sector plate and circular sector plate are shown in Figure 11. In this analysis, the inner-to-outer radii ratios $R_{0} / R_{1}=0.5$ for annular sector plate and $R_{0} / R_{1}=0$ for circular sector plate are used, and these sector annular angles gradually increase from $2^{\circ}$ to $358^{\circ}$. For sector annular plates with CCCC, FCFC, and CCCF boundary conditions and circular sector plates with CCC, CFC, and CCF boundary conditions, the conclusion can be clearly obtained that the frequency parameters of the plate are in the monotonically decreasing state when the sector angle increases. The frequency parameters sharply decrease 

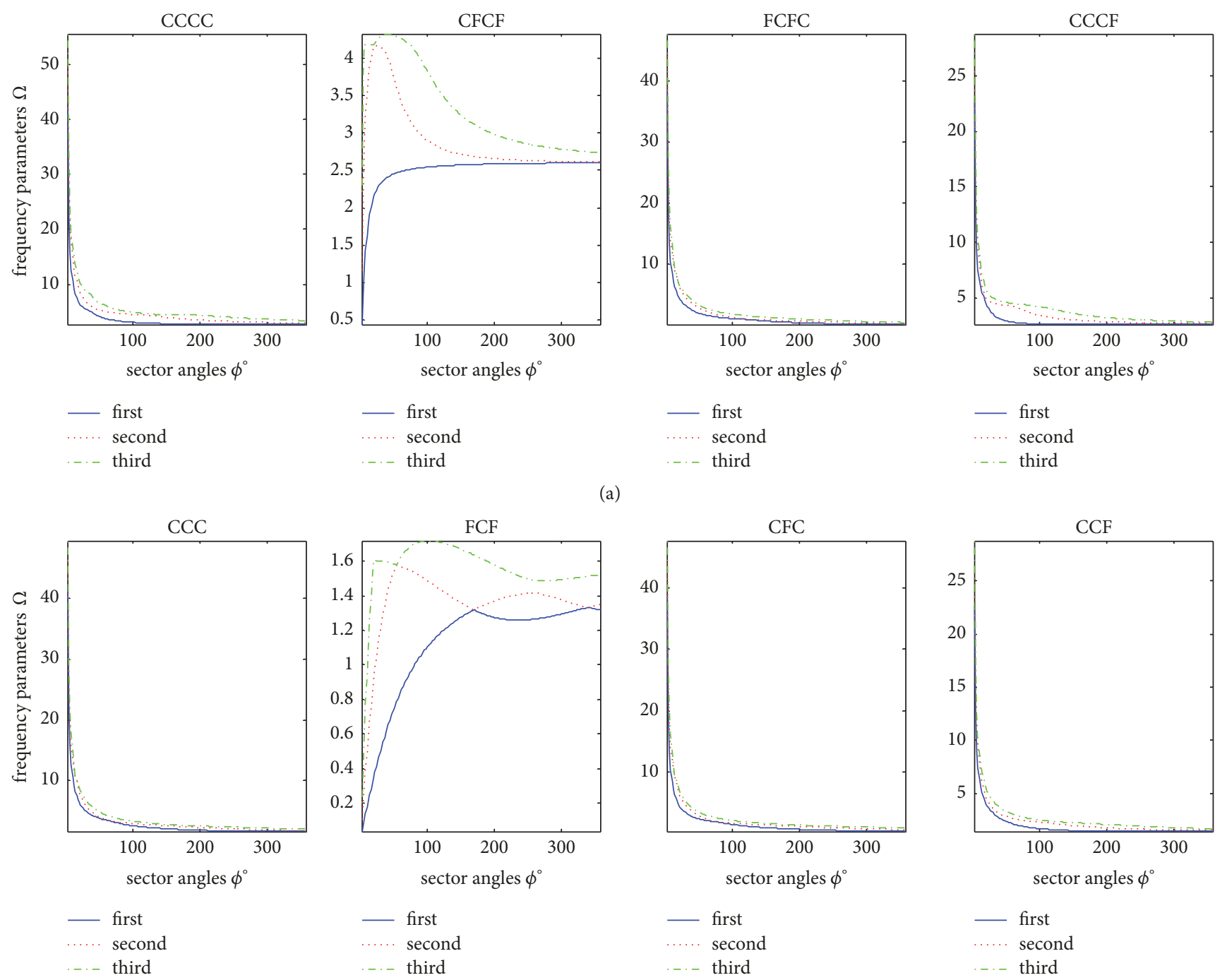

(b)

FIGURE 11: The variations of some frequency parameters for sector plates with different boundary conditions and sector angles: (a) annular sector plate; (b) circular sector plate.

before the annular sector angle is small, that is, $\phi \leq 10^{\circ}$, and the descending velocities of the frequency parameters become slower when the sector angle varies from $10^{\circ}$ to $100^{\circ}$, and the frequency parameters remain stable after the annular sector angle $\phi \geq 100^{\circ}$. For the cases of CFCF annular sector plate and FCF circular sector plate, the frequency parameters increase rapidly with beginning and then decease or increase slightly with sector angle increasing in general. The effects of inner-to-outer radii ratios on first three natural frequencies of annular sector plate and annular plate are illustrated in Figure 12. The sector angle $60^{\circ}$ is considered for annular sector plate. For the case of sector annular plate under boundary conditions CCCC, CFCF, and CCCF and annular plates under boundary conditions $\mathrm{CC}, \mathrm{CF}$, and $\mathrm{FC}$, the frequency parameters always increase and the ascending velocities of frequency parameters gradually become faster as the inner-to-outer radii ratios increase. For the FCFC sector annular plate, the frequency parameters always decrease. Figure 13 presents the variational rules of first three frequency parameters for the annular sector plate by changing the inner-to-outer radii ratios. The various sector angles and boundary conditions are considered in this analysis. It is obvious that the changes of natural frequencies for CCCC, CFCF, and CCCF boundary conditions also increase as the inner-to-outer radii ratios increase. But for FCFC boundary condition the variations of frequency parameter are more complicated; when the sector angle is larger than $10^{\circ}$, the first three frequency parameters decrease with the increasing inner-to-outer radii ratios. When the sector angle is smaller $10^{\circ}$, the variations of frequency parameters are different. For instance, the first frequency parameter monotonically decreases, the second frequency parameter first decreases and 


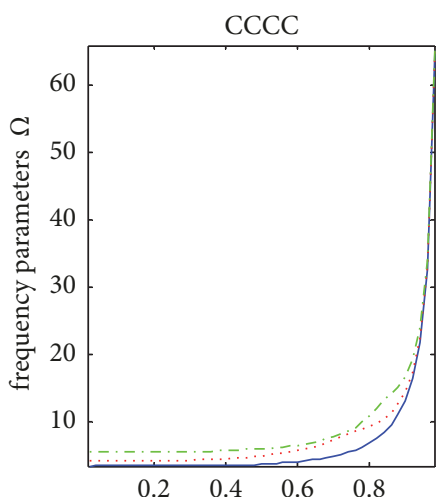

inner-to-outer radii ratios $R_{0} / R_{1}$

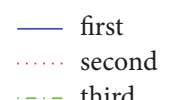

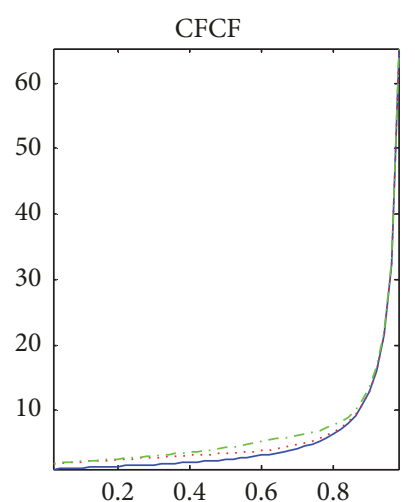

inner-to-outer radii ratios $R_{0} / R_{1}$

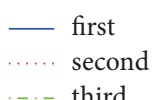

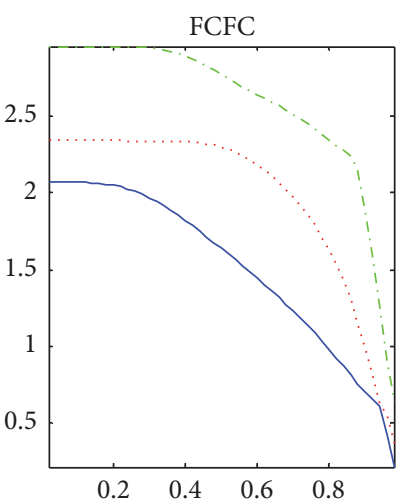

inner-to-outer radii ratios $R_{0} / R_{1}$

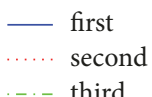

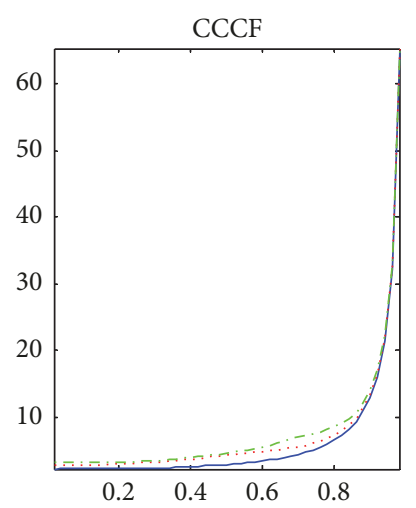

inner-to-outer radii ratios $R_{0} / R_{1}$

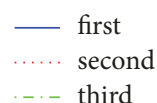

(a)
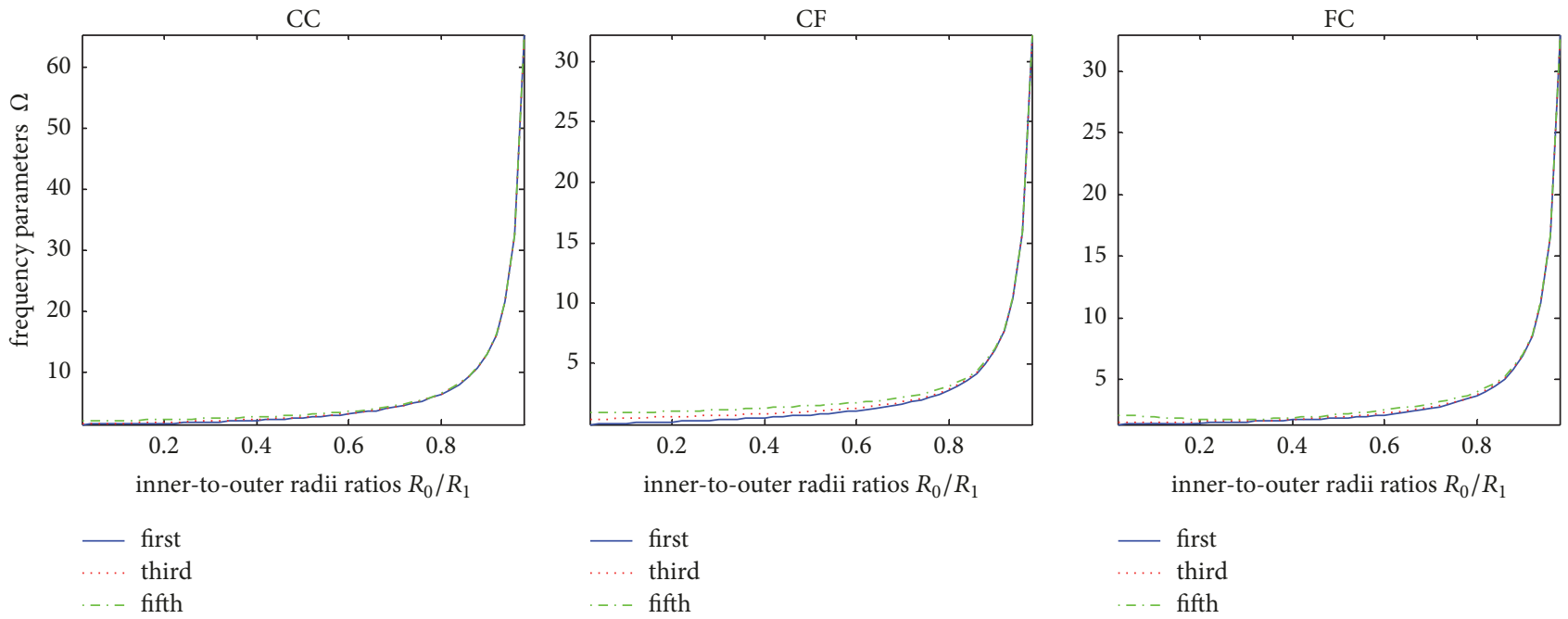

(b)

Figure 12: The variations of some frequency parameters for plates with different boundary conditions and inner-to-outer radii ratios: (a) annular sector plate; (b) annular plate.

then increases, and there are almost few changes in the third frequency parameters.

\section{Conclusions}

In this paper, the free in-plane vibration of annular sector, annular, circular sector, and circular plates is investigated by an effective approximate formulation based on isogeometric finite element analysis. By using the NURBS basic functions in this method, the polynomial order can be easily evaluated and the desired geometry can be obtained. The gap between CAD and FEA can be possibly narrowed by applying the identical functions to construct the unknown filed and the geometry. The in-plane vibration differential equations for sector plate, annular plate, and circular plate can be systematically derived by the principle of virtual work. Three refinement schemes (the $h$-, $p^{-}$, and $k$-refinement strategies) are used to discretize the geometry and refine element, and the efficiency and rapid convergence of the $k$-refinement in IGA have been confirmed. Then several numerical results of the natural nondimensional frequencies for sector, annular, and circular plates are computed and compared with relevant data in open references or from finite element method. It can be easily obtained that the current formulation provides a reliability means for the in-plane vibration of these plates. Additionally, some new natural nondimensional frequency parameters and corresponding mode shapes for annular plate, circular plate, and sector plates are presented. The new calculated results can be adopted by other researchers as a benchmark for future researches. The effects of sector angle and ratio of inside to outside radii for these plates with different boundary conditions are investigated as well. 

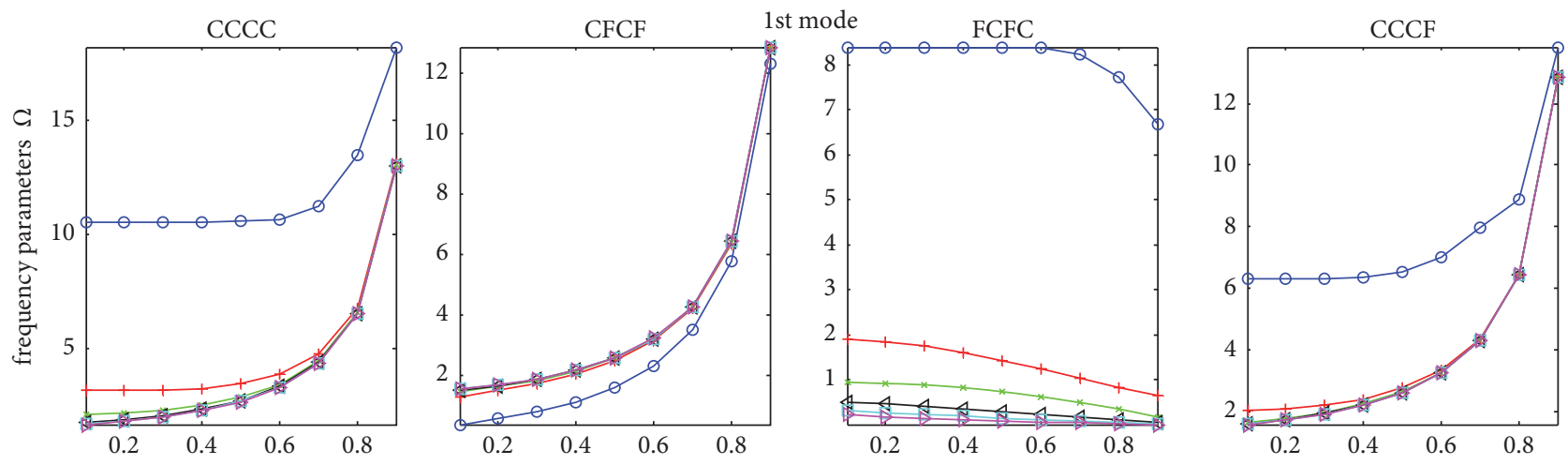

inner-to-outer radii ratios $R_{0} / R_{1}$ inner-to-outer radii ratios $R_{0} / R_{1}$

inner-to-outer radii ratios $R_{0} / R_{1}$

inner-to-outer radii ratios $R_{0} / R_{1}$

\begin{tabular}{|c|c|c|c|c|c|c|c|}
\hline$-10^{\circ}$ & $\neg 220^{\circ}$ & $-10^{\circ}$ & $4220^{\circ}$ & $-10^{\circ}$ & $\neg 220^{\circ}$ & $-10^{\circ}$ & $4220^{\circ}$ \\
\hline $1-70^{\circ}$ & $\square-290^{\circ}$ & $+70^{\circ}$ & $\square-290^{\circ}$ & $+70^{\circ}$ & $\square-290^{\circ}$ & $170^{\circ}$ & $\square-290^{\circ}$ \\
\hline$\approx 140^{\circ}$ & $\rightarrow 350^{\circ}$ & $-140^{\circ}$ & $\rightarrow \quad 350^{\circ}$ & $-140^{\circ}$ & $\rightarrow 350^{\circ}$ & $\because 140^{\circ}$ & $\rightarrow$ \\
\hline
\end{tabular}
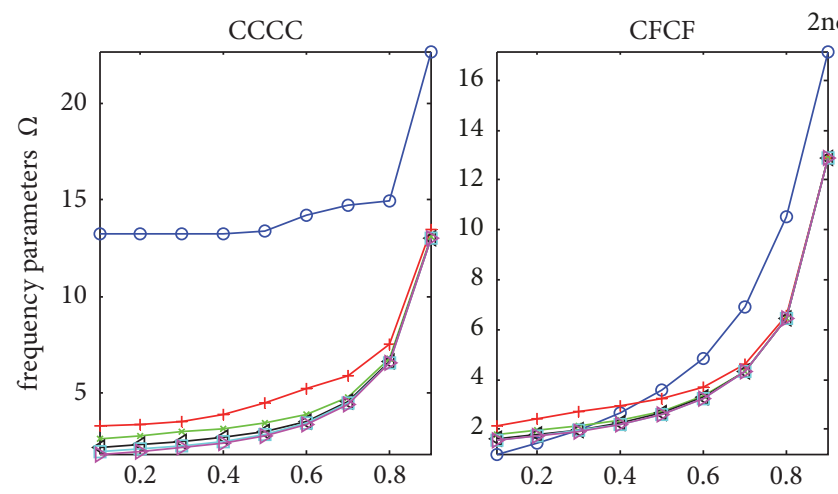

2nd mode
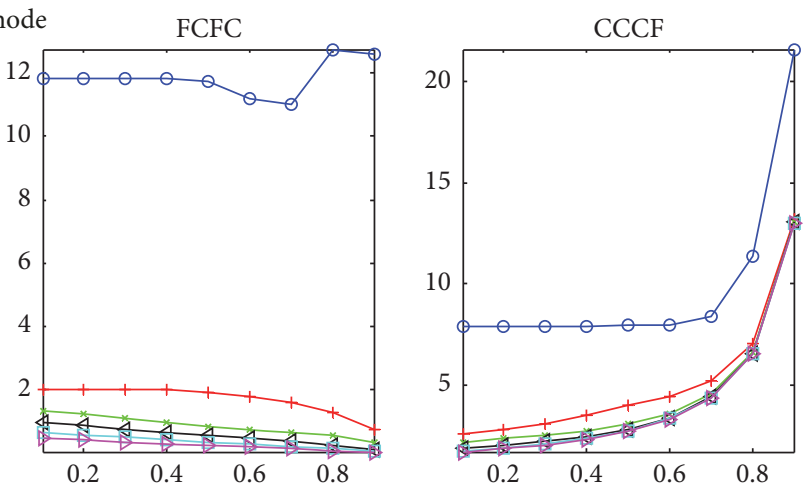

inner-to-outer radii ratios $R_{0} / R_{1}$ inner-to-outer radii ratios $R_{0} / R_{1}$

inner-to-outer radii ratios $R_{0} / R_{1}$

inner-to-outer radii ratios $R_{0} / R_{1}$

\begin{tabular}{|c|c|c|c|c|c|c|c|}
\hline$\multimap 10^{\circ}$ & $\neg 220^{\circ}$ & $-0-10^{\circ}$ & $\dashv 220^{\circ}$ & $-10^{\circ}$ & $\neg 220^{\circ}$ & $-10^{\circ}$ & $\neg 220$ \\
\hline$千 70^{\circ}$ & $\square \quad 290^{\circ}$ & $千 70^{\circ}$ & $\square \quad 290^{\circ}$ & $\div 70^{\circ}$ & $\square \quad 290^{\circ}$ & $千 70^{\circ}$ & $\square \quad 290$ \\
\hline$\approx 140^{\circ}$ & $\rightarrow 350^{\circ}$ & $-\approx-140^{\circ}$ & $\triangleright \quad 350^{\circ}$ & $\approx 140^{\circ}$ & $\rightarrow$ & $\approx 140^{\circ}$ & $\rightarrow-350$ \\
\hline
\end{tabular}
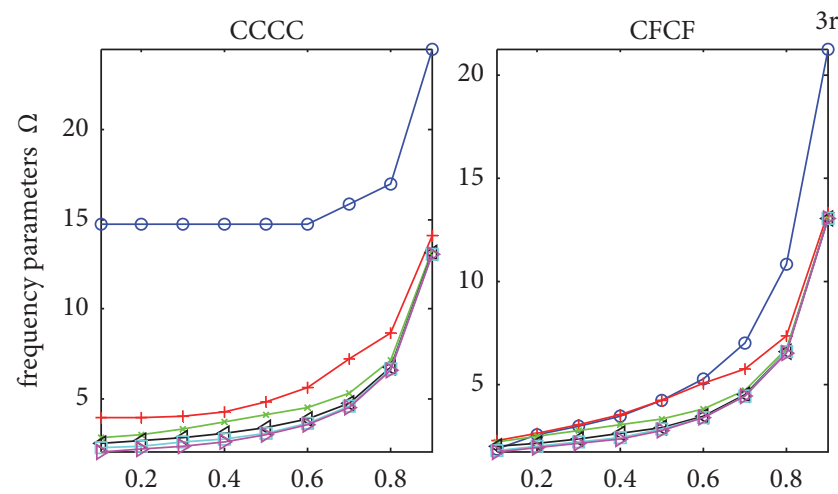

3rd mode
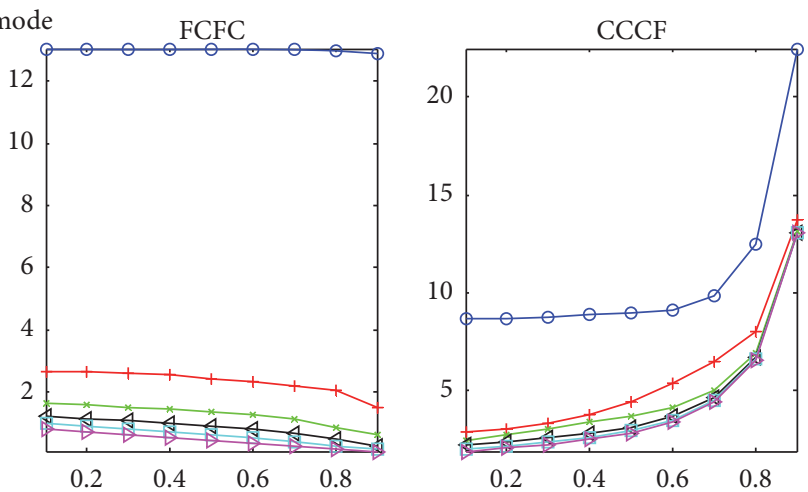

inner-to-outer radii ratios $R_{0} / R_{1}$ inner-to-outer radii ratios $R_{0} / R_{1}$

inner-to-outer radii ratios $R_{0} / R_{1}$

inner-to-outer radii ratios $R_{0} / R_{1}$

$$
\begin{aligned}
& -10^{\circ} \quad-4220^{\circ} \\
& \begin{array}{lll}
- & 10^{\circ} \quad \triangleleft 220^{\circ}
\end{array} \\
& +70^{\circ}-\square 290^{\circ} \\
& \begin{array}{lll}
- & 10^{\circ} \quad \varangle 220^{\circ}
\end{array} \\
& +70^{\circ} \rightarrow 290^{\circ} \\
& \begin{array}{lll}
- & 10^{\circ} \quad \varangle 220^{\circ}
\end{array} \\
& +70^{\circ} \\
& \longrightarrow 140^{\circ} \rightarrow 350^{\circ} \\
& \longrightarrow 140^{\circ} \\
& \rightarrow 350^{\circ} \\
& \multimap 140^{\circ} \\
& \rightarrow 350^{\circ} \\
& \longrightarrow 140^{\circ} \\
& \rightarrow 350^{\circ}
\end{aligned}
$$

FIGURE 13: The variation of first three frequency parameters for the annular sector plate with different boundary conditions, inner-to-outer radii ratios, and sector angles. 


\section{Conflicts of Interest}

The authors declare that they have no conflicts of interest.

\section{Acknowledgments}

This paper is supported by the National Natural Science Foundation of China (nos. 51775125 and 51279035) and the Fundamental Research Funds for the Central Universities of China (no. HEUCFG201713).

\section{References}

[1] T. Ye, G. Jin, Z. Su, and X. Jia, "A unified Chebyshev-Ritz formulation for vibration analysis of composite laminated deep open shells with arbitrary boundary conditions," Archive of Applied Mechanics, vol. 84, no. 4, pp. 441-471, 2014.

[2] G. Jin, Z. Su, T. Ye, and X. Jia, "Three-dimensional vibration analysis of isotropic and orthotropic conical shells with elastic boundary restraints," International Journal of Mechanical Sciences, vol. 89, pp. 207-221, 2014.

[3] G. Y. Jin, T. Ye, X. L. Ma, Y. Chen, Z. Su, and X. Xie, "A unified approach for the vibration analysis of moderately thick composite laminated cylindrical shells with arbitrary boundary conditions," International Journal of Mechanical Sciences, vol. 75, pp. 357-376, 2013.

[4] T. Ye, G. Jin, Z. Su, and Y. Chen, "A modified Fourier solution for vibration analysis of moderately thick laminated plates with general boundary restraints and internal line supports," International Journal of Mechanical Sciences, vol. 80, pp. 29-46, 2014.

[5] T. Ye, G. Jin, and Z. Su, “Three-dimensional vibration analysis of laminated functionally graded spherical shells with general boundary conditions," Composite Structures, vol. 116, no. 1, pp. 571-588, 2014.

[6] Y. F. Xing and B. Liu, "Exact solutions for the free in-plane vibrations of rectangular plates," International Journal of Mechanical Sciences, vol. 51, no. 3, pp. 246-255, 2009.

[7] A. N. Bercin and R. S. Langley, "Application of the dynamic stiffness technique to the in-plane vibrations of plate structures," Computers \& Structures, vol. 59, no. 5, pp. 869-875, 1996.

[8] M. Nefovska-Danilovic and M. Petronijevic, "In-plane free vibration and response analysis of isotropic rectangular plates using the dynamic stiffness method," Computers \& Structures, vol. 152, pp. 82-95, 2015.

[9] D. J. Gorman, "Free in-plane vibration analysis of rectangular plates by the method of superposition," Journal of Sound and Vibration, vol. 272, no. 3-5, pp. 831-851, 2004.

[10] D. J. Gorman, "Exact solutions for the free in-plane vibration of rectangular plates with two opposite edges simply supported," Journal of Sound and Vibration, vol. 294, no. 1-2, pp. 131-161, 2006.

[11] I. V. Andrianov, J. Awrejcewicz, and V. Chernetskyy, "Analysis of natural in-plane vibration of rectangular plates using homotopy perturbation approach," Mathematical Problems in Engineering, vol. 2006, Article ID 20598, 8 pages, 2006.

[12] Y. Chen, G. Jin, and Z. Liu, "Flexural and in-plane vibration analysis of elastically restrained thin rectangular plate with cutout using Chebyshev-Lagrangian method," International Journal of Mechanical Sciences, vol. 89, pp. 264-278, 2014.
[13] A. H. Mohazzab and L. Dozio, "A spectral collocation solution for in-plane eigenvalue analysis of skew plates," International Journal of Mechanical Sciences, vol. 94-95, pp. 199-210, 2015.

[14] Y. Zhou, Q. Wang, D. Shi, Q. Liang, and Z. Zhang, "Exact solutions for the free in-plane vibrations of rectangular plates with arbitrary boundary conditions," International Journal of Mechanical Sciences, vol. 130, pp. 1-10, 2017.

[15] Z. Shi, X. Yao, F. Pang, and Q. Wang, "A semi-analytical solution for in-plane free vibration analysis of functionally graded carbon nanotube reinforced composite circular arches with elastic restraints," Composite Structures, vol. 182, pp. 420-434, 2017.

[16] H. Rostami, A. Rahbar Ranji, and F. Bakhtiari-Nejad, "Free in-plane vibration analysis of rotating rectangular orthotropic cantilever plates," International Journal of Mechanical Sciences, vol. 115-116, pp. 438-456, 2016.

[17] H. Li, F. Pang, X. Wang, and S. Li, "Benchmark Solution for Free Vibration of Moderately Thick Functionally Graded Sandwich Sector Plates on Two-Parameter Elastic Foundation with General Boundary Conditions," Shock and Vibration, vol. 2017, Article ID 4018629, 35 pages, 2017.

[18] D. T. Huang, "Effects of constraint, circular cutout and in-plane loading on vibration of rectangular plates," International Journal of Mechanical Sciences, vol. 68, pp. 114-124, 2013.

[19] T. Irie, G. Yamada, and Y. Muramoto, "Natural frequencies of in-plane vibration of annular plates," Journal of Sound and Vibration, vol. 97, no. 1, pp. 171-175, 1984.

[20] S. Bashmal, R. Bhat, and S. Rakheja, "In-plane free vibration of circular annular disks," Journal of Sound and Vibration, vol. 322, no. 1-2, pp. 216-226, 2009.

[21] N. H. Farag and J. Pan, "Modal characteristics of in-plane vibration of circular plates clamped at the outer edge," The Journal of the Acoustical Society of America, vol. 113, no. 4 I, pp. 1935-1946, 2003.

[22] S. A. Eftekhari, "Differential quadrature procedure for in-plane vibration analysis of variable thickness circular arches traversed by a moving point load," Applied Mathematical Modelling, vol. 40, no. 7-8, pp. 4640-4663, 2016.

[23] A. V. Singh and T. Muhammad, "Free in-plane vibration of isotropic non-rectangular plates," Journal of Sound and Vibration, vol. 273, no. 1-2, pp. 219-231, 2004.

[24] I. P. Chan, "Frequency equation for the in-plane vibration of a clamped circular plate," Journal of Sound and Vibration, vol. 313, no. 1-2, pp. 325-333, 2008.

[25] X. Shi, W. Li, and D. Shi, "Free in-plane vibrations of annular sector plates with elastic boundary supports," in Proceedings of the 164th Meeting of the Acoustical Society of America 2012, October 2012.

[26] Q. Wang, D. Shi, Q. Liang, and F. e Ahad, "A unified solution for free in-plane vibration of orthotropic circular, annular and sector plates with general boundary conditions," Applied Mathematical Modelling, vol. 40, no. 21-22, pp. 9228-9253, 2016.

[27] T. J. Hughes, J. A. Cottrell, and Y. Bazilevs, "Isogeometric analysis: CAD, finite elements, NURBS, exact geometry and mesh refinement," Computer Methods Applied Mechanics and Engineering, vol. 194, no. 39-41, pp. 4135-4195, 2005.

[28] L. A. Piegl and W. Tiller, The NURBS Book, Springer, Berlin, Germany, 1995.

[29] J. A. Cottrell, A. Reali, Y. Bazilevs, and T. . Hughes, "Isogeometric analysis of structural vibrations," Computer Methods Applied Mechanics and Engineering, vol. 195, no. 41-43, pp. 5257-5296, 2006. 
[30] S. Yin, J. S. Hale, T. Yu, T. Q. Bui, and S. P. A. Bordas, "Isogeometric locking-free plate element: A simple first order shear deformation theory for functionally graded plates," Composite Structures, vol. 118, no. 1, pp. 121-138, 2014.

[31] T. J. Hughes, A. Reali, and G. Sangalli, "Efficient quadrature for NURBS-based isogeometric analysis," Computer Methods Applied Mechanics and Engineering, vol. 199, no. 5-8, pp. 301313, 2010.

[32] J. Kiendl, K.-U. Bletzinger, J. Linhard, and R. Wüchner, "Isogeometric shell analysis with Kirchhoff-Love elements," Computer Methods Applied Mechanics and Engineering, vol. 198, no. 49-52, pp. 3902-3914, 2009.

[33] S. Shojaee, N. Valizadeh, E. Izadpanah, T. Bui, and T.-V. Vu, "Free vibration and buckling analysis of laminated composite plates using the NURBS-based isogeometric finite element method," Composite Structures, vol. 94, no. 5, pp. 1677-1693, 2012.

[34] S. Cho and S.-H. Ha, "Isogeometric shape design optimization: exact geometry and enhanced sensitivity," Structural and Multidisciplinary Optimization, vol. 38, no. 1, pp. 53-70, 2009.

[35] W. A. Wall, M. A. Frenzel, and C. Cyron, "Isogeometric structural shape optimization," Computer Methods Applied Mechanics and Engineering, vol. 197, no. 33-40, pp. 2976-2988, 2008.

[36] Y. Zhang, Y. Bazilevs, S. Goswami, C. L. Bajaj, and T. J. Hughes, "Patient-specific vascular NURBS modeling for isogeometric analysis of blood flow," Computer Methods Applied Mechanics and Engineering, vol. 196, no. 29-30, pp. 2943-2959, 2007.

[37] Y. Bazilevs, V. M. Calo, Y. Zhang, and T. J. R. Hughes, "Isogeometric fluid-structure interaction analysis with applications to arterial blood flow," Computational Mechanics, vol. 38, no. 4-5, pp. 310-322, 2006.

[38] R. Kruse, N. Nguyen-Thanh, L. De Lorenzis, and T. J. Hughes, "Isogeometric collocation for large deformation elasticity and frictional contact problems," Computer Methods Applied Mechanics and Engineering, vol. 296, pp. 73-112, 2015.

[39] J. Kiendl, M.-C. Hsu, M. C. Wu, and A. Reali, "Isogeometric Kirchhoff-Love shell formulations for general hyperelastic materials," Computer Methods Applied Mechanics and Engineering, vol. 291, pp. 280-303, 2015.

[40] A. B. Tepole, H. Kabaria, K.-U. Bletzinger, and E. Kuhl, "Isogeometric Kirchhoff-Love shell formulations for biological membranes," Computer Methods Applied Mechanics and Engineering, vol. 293, pp. 328-347, 2015.

[41] D. J. Benson, Y. Bazilevs, M. C. Hsu, and T. . Hughes, "Isogeometric shell analysis: the Reissner-Mindlin shell," Computer Methods Applied Mechanics and Engineering, vol. 199, no. 5-8, pp. 276-289, 2010.

[42] D. J. Benson, Y. Bazilevs, M.-C. Hsu, and T. . Hughes, "A large deformation, rotation-free, isogeometric shell," Computer Methods Applied Mechanics and Engineering, vol. 200, no. 13-16, pp. 1367-1378, 2011.

[43] L. V. Tran, A. J. M. Ferreira, and H. Nguyen-Xuan, "Isogeometric analysis of functionally graded plates using higher-order shear deformation theory," Composites Part B: Engineering, vol. 51, no. 4, pp. 368-383, 2013.

[44] L. V. Tran, H. A. Ly, J. Lee, M. A. Wahab, and H. NguyenXuan, "Vibration analysis of cracked FGM plates using higherorder shear deformation theory and extended isogeometric approach," International Journal of Mechanical Sciences, vol. 9697, pp. 65-78, 2015.

[45] H. Nguyen-Xuan, L. V. Tran, C. H. Thai, S. Kulasegaram, and S. P. A. Bordas, "Isogeometric analysis of functionally graded plates using a refined plate theory," Composites Part B: Engineering, vol. 64, pp. 222-234, 2014.

[46] C. H. Thai, A. J. M. Ferreira, E. Carrera, and H. Nguyen-Xuan, "Isogeometric analysis of laminated composite and sandwich plates using a layerwise deformation theory," Composite Structures, vol. 104, pp. 196-214, 2013.

[47] C. H. Thai, A. Ferreira, S. Bordas, T. Rabczuk, and H. NguyenXuan, "Isogeometric analysis of laminated composite and sandwich plates using a new inverse trigonometric shear deformation theory," European Journal of Mechanics - A-Solids, vol. 43, no. 1, pp. 89-108, 2014.

[48] J. N. Reddy, Energy Principles And Variational Methods in Applied Mechanics, John Wiley \& Sons Inc., New York, NY, USA, 1984.

[49] J. N. Reddy, "A simple higher order theory for laminated composite plates," Journal of Applied Mechanics, vol. 51, no. 4, pp. 745-752, 1984.

[50] J. A. Cottrell, T. I. R. Hughes, and Y. Bazilevs, Isogeometric Analysis: toward Integration of CAD and FEM, John Wiley \& Sons Inc., New York, NY, USA, 2009.

[51] R. B. Lehoucq and D. C. Sorensen, "Deflation techniques for an implicitly restarted Arnoldi iteration," SIAM Journal on Matrix Analysis and Applications, vol. 17, no. 4, pp. 789-821, 1996.

[52] D. C. Sorensen, "Implicit application of polynomial filters in a $k$-step Arnoldi method," SIAM Journal on Matrix Analysis and Applications, vol. 13, no. 1, pp. 357-385, 1992.

[53] J. A. Cottrell, T. J. R. Hughes, and A. Reali, "Studies of refinement and continuity in isogeometric structural analysis," Computer Methods Applied Mechanics and Engineering, vol. 196, no. 41-44, pp. 4160-4183, 2007. 


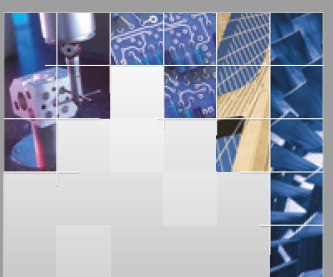

\section{Enfincering}
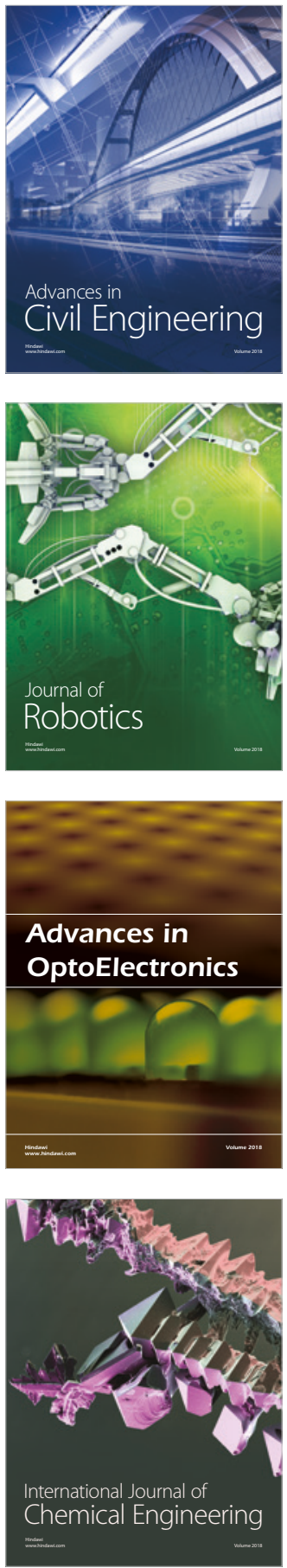

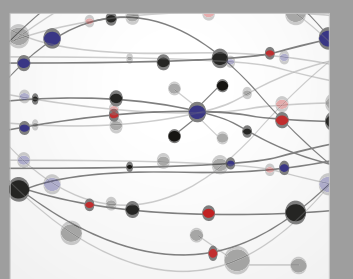

\section{Rotating \\ Machinery}

The Scientific World Journal

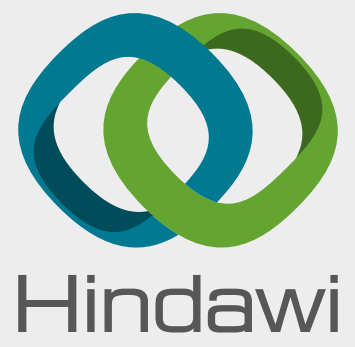

Submit your manuscripts at

www.hindawi.com
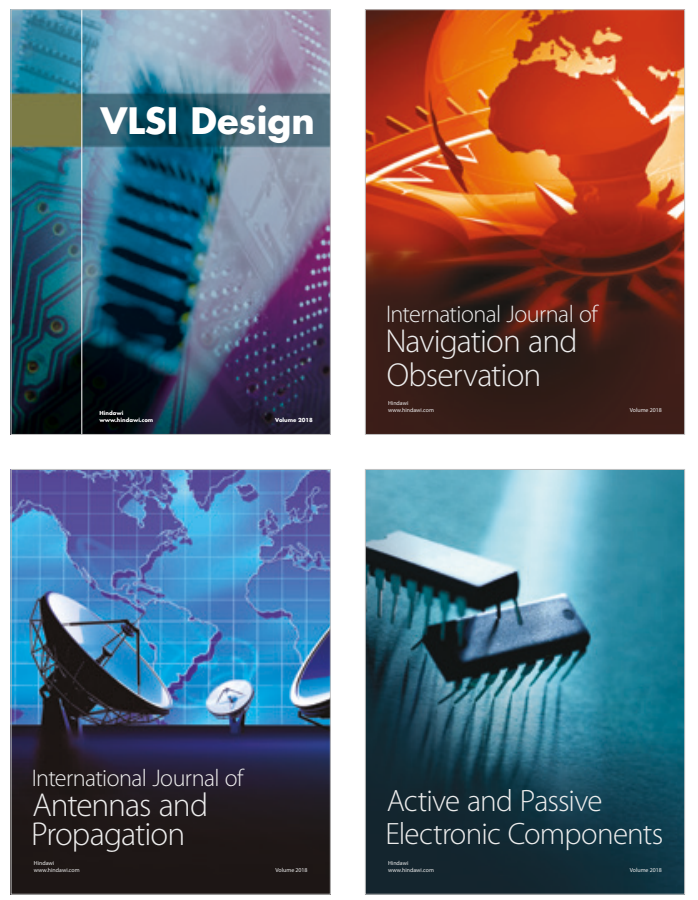
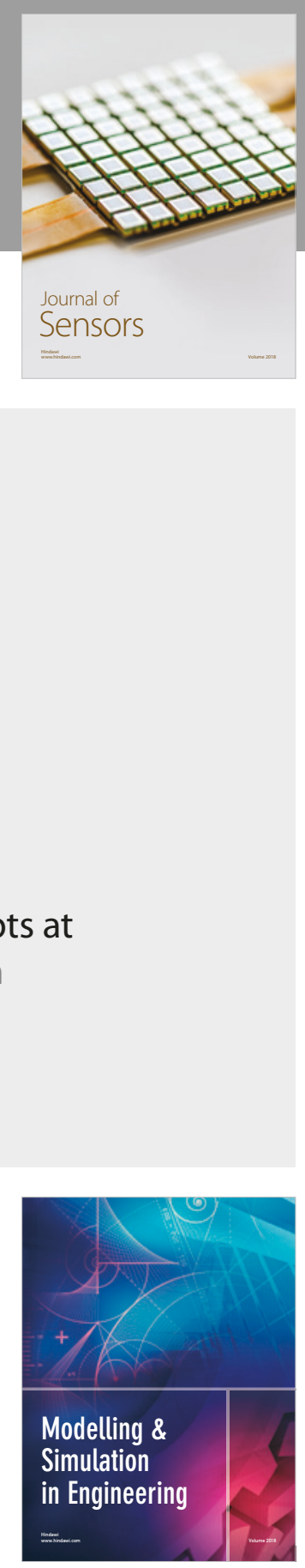

\section{Advances \\ Multimedia}
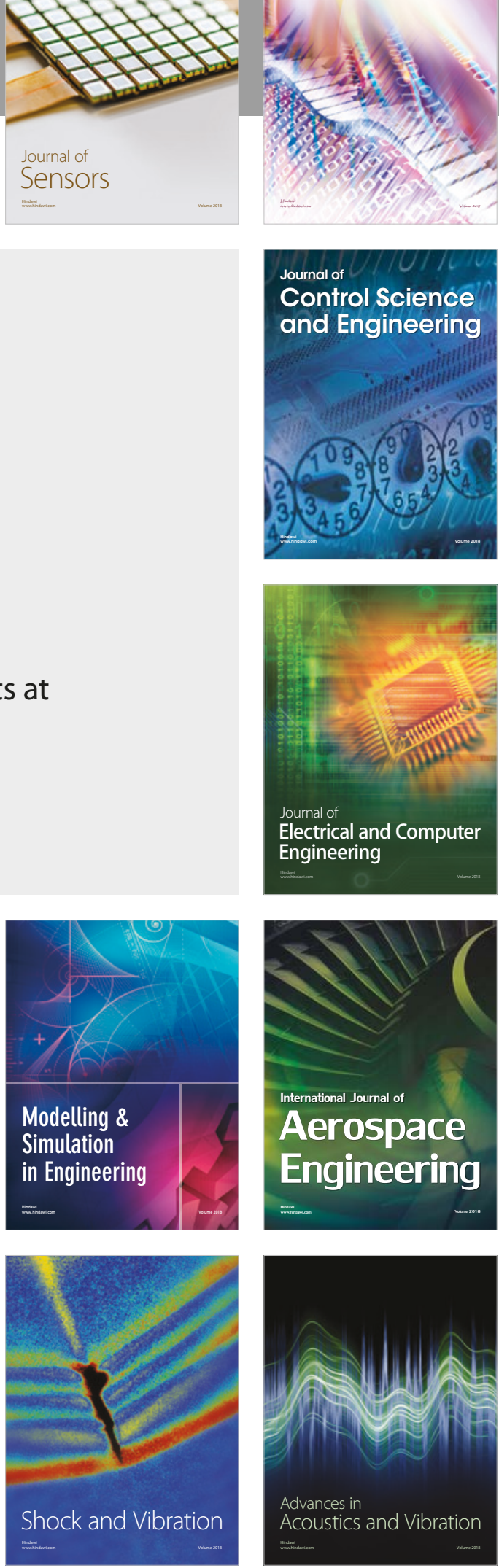